\title{
Duality for multiobjective variational control problems with $(\Phi, \rho)$-invexity
}

\author{
Tadeusz Antczak
}

Received: 12 November 2012 / Accepted: 29 May 2013 / Published online: 29 November 2013 C The Author(s) 2013. This article is published with open access at Springerlink.com

\begin{abstract}
In this paper, Mond-Weir and Wolfe type duals for multiobjective variational control problems are formulated. Several duality theorems are established relating efficient solutions of the primal and dual multiobjective variational control problems under $(\Phi, \rho)$-invexity. The results generalize a number of duality results previously established for multiobjective variational control problems under other generalized convexity assumptions.
\end{abstract}

Keywords Multiobjective variational problems · Efficient solution · $(\Phi, \rho)$-invexity $\cdot$ Duality

Mathematics Subject Classification (2000) $\quad 65 \mathrm{~K} 10 \cdot 90 \mathrm{C} 29 \cdot 90 \mathrm{C} 46 \cdot 26 \mathrm{~B} 25$

\section{Introduction}

Duality constitute an essential part of study of mathematical programming in the sense that these lay down the foundation of algorithms for a solution of an optimization problem. In recent years, duality theory for multiobjective variational problems has been of much interest, and several contributions have been made to its development. This is also a consequence of its applications in physics, economics, management sciences, engineering problems, etc.

Various generalized convexity notions have been used recently to prove duality results for a larger class of variational control problems than those ones with convex functions. Mond et al. [14] extended the concept of invexity introduced by Hanson [8] to the continuous case and used it to generalize earlier duality results for a class

T. Antczak $(\bowtie)$

Faculty of Mathematics and Computer Science, University of Łódź,

Banacha 22, 90-238 Łódź, Poland

e-mail: antczak@math.uni.lodz.pl 
of variational problems. Mond and Smart [15] extended the duality theorems for a class of static nondifferentiable problems with Wolfe type and Mond-Weir type duals, and further extended these for the continuous analogues. In 1992, Bector and Husain [2] first applied duality method of ordinary multiobjective optimization problem to multiobjective variational problem, and obtained duality results for properly efficient solution under convexity assumptions on involved functions. Nahak and Nanda formulated Wolfe and Mond-Weir type duals for multiobjective variational problems and proved several duality theorems under invexity assumptions [17] and also under pseudo-invexity assumptions [19]. Bhatia and Kumar [3] have studied multiobjective control problems under $\rho$-pseudoinvexity, $\rho$-strictly pseudoinvexity, $\rho$-quasiinvexity, strictly $\rho$-quasiinvexity assumptions. They derived duality theorems for multiobjective control problems under generalized invexity notions mentioned above. Chen [7] discussed and formulated Wolfe and Mond-Weir type duals for a class of nondifferentiable multiobjective variational problems. Under invexity assumptions on the objective and the constraint functions, he proved weak and strong duality theorems to related properly efficient solutions for primal and dual multiobjective control problems. Nahak [16] formulated Wolfe and Mond-Weir type duals for multiobjective control problems and, under pseudo-invexity/quasi-invexity, proved weak and strong duality theorems to relate efficient solutions of the primal and dual problems. Mishra and Mukherjee [10] generalized $(F, \rho)$-convexity introduced by Preda [20], and established duality results for a class of multiobjective variational control problems with $(F, \rho)$-convex functions. Nahak and Nanda [18] used the concept of efficiency to formulate Wolfe and Mond-Weir type duals for multiobjective variational control problems and established weak and strong duality theorems under generalized $(F, \rho)$ convexity assumptions. Also Reddy and Mukherjee [21] have studied duality theorems under $(F, \rho)$-convexity assumptions and related efficient solutions of the primal and dual problems for multiobjective fractional control problems. In [11], Mishra and Mukherjee obtained Mond-Weir-type duality results for multiobjective control problems under $V$-invexity assumptions and their generalizations. Bhatia and Mehra [4] extended the concepts of $B$-type I and generalized $B$-type I functions to the continuous case and they used these concepts to establish sufficient optimality conditions and duality results for multiobjective variational programming problems. Under the assumption of invexity and its generalization, Xiuhong [23] proved duality theorems through a parametric approach to related properly efficient solutions of the primal and dual multiobjective control problems. Kim and Kim [9] introduced new classes of generalized $V$-type I invex functions for multiobjective variational problems and obtained duality results for Mond-Weir type duals under the generalized $V$-type I invexity assumptions and their generalizations. In [24], Zhian and Qingkai discussed duality for multiobjective control problems with the same objective functionals and constraint conditions as in [3], but with the invexity defined in [15]. Ahmad and Sharma [1] extended the generalized $(F, \alpha, \rho, \theta)-V$-convex functions to multiobjective variational control problems and proved Wolfe type and Mond Weir type duality results for multiobjective variational control programming problems with these functions.

In [6], for scalar differentiable optimization problems, Caristi et al. [6] introduced $(\Phi, \rho)$-invexity notion. They proved optimality and duality results for smooth scalar optimization problems with $(\Phi, \rho)$-invex functions. 
In this paper, the notion of scalar $(\Phi, \rho)$-invexity is extended to the continuous case and it is defined for multiobjective variational control problems. By utilizing this concept of generalized convexity, we prove Mond Weir type and Wolfe type duality results for multiobjective variational control programming problem involving $(\Phi, \rho)$ invex functions with respect to, not necessarily, the same $\rho$ (with the exception of those the equality constraints for which the associated piecewise smooth functions satisfying the constraints of duals are negative). Thus, we generalize Mond Weir type and Wolfe type duality results for multiobjective variational control programming problems established in the literature under other generalized convexity notions.

\section{Preliminaries, notations and statement of the problem}

The following convention for equalities and inequalities will be used in the paper.

For any $x=\left(x_{1}, x_{2}, \ldots, x_{n}\right)^{T}, y=\left(y_{1}, y_{2}, \ldots, y_{n}\right)^{T}$, we define:

(i) $x=y$ if and only if $x_{i}=y_{i}$ for all $i=1,2, \ldots, n$;

(ii) $x>y$ if and only if $x_{i}>y_{i}$ for all $i=1,2, \ldots, n$;

(iii) $x \geqq y$ if and only if $x_{i} \geqq y_{i}$ for all $i=1,2, \ldots, n$;

(iv) $x \geq y$ if and only if $x \geqq y$ and $x \neq y$.

Let $I=[a, b]$ be a real interval and let $A=\{1,2, \ldots, p\}, J=\{1,2, \ldots, q\}$ and $K=\{1, \ldots, s\}$.

In this paper, we assume that $x(t)$ is an $n$-dimensional piecewise smooth function of $t$, and $\dot{x}(t)$ is the derivative of $x(t)$ with respect to $t$ in $[a, b]$.

Denote by $X$ the space of piecewise smooth state functions $x: I \rightarrow R^{n}$ with norm $\|x\|=\|x\|_{\infty}+\|D x\|_{\infty}$, where the differentiation operator $D$ is given by $z=D x \Longleftrightarrow x(t)=x(a)+\int_{a}^{t} z(s) d s$, where $x(a)$ is a given boundary value. Therefore $\frac{d}{d t} \equiv D$ except at discontinuities.

In the paper, consider the following multiobjective variational control problem (MVPP):

$$
\begin{gathered}
\text { Minimize } \int_{a}^{b} f(t, x(t), \dot{x}(t)) d t \\
=\left(\int_{a}^{b} f_{1}(t, x(t), \dot{x}(t)), \ldots, \int_{a}^{b} f_{p}(t, x(t), \dot{x}(t))\right) \\
\text { subject to } g(t, x(t), \dot{x}(t)) \leqq 0, t \in I, \\
h(t, x(t), \dot{x}(t))=0, t \in I, \\
x(a)=\alpha, x(b)=\beta,
\end{gathered}
$$

where $f=\left(f_{1}, \ldots, f_{p}\right): I \times R^{n} \times R^{n} \rightarrow R^{p}$, is a $p$-dimensional function and each its component is a continuously differentiable real scalar function, $g=\left(g_{1}, \ldots, g_{q}\right)$ : 
$I \times R^{n} \times R^{n} \rightarrow R^{q}$ and $h=\left(h_{1}, \ldots, h_{s}\right): I \times R^{n} \times R^{n} \rightarrow R^{s}$ are assumed to be continuously differentiable $q$-dimensional and $s$-dimensional functions, respectively.

For notational simplicity, we write $x(t)$ and $\dot{x}(t)$ as $x$ and $\dot{x}$, respectively. We denote the partial derivatives of $f^{1}$ with respect to $t, x$ and $\dot{x}$, respectively, by $f_{t}^{1}, f_{x}^{1}, f_{\dot{x}}^{1}$ such that $f_{x}^{1}=\left(\frac{\partial f^{1}}{\partial x_{1}}, \ldots, \frac{\partial f^{1}}{\partial x_{n}}\right)$ and $f_{\dot{x}}^{1}=\left(\frac{\partial f^{1}}{\partial \dot{x}_{1}}, \ldots, \frac{\partial f^{1}}{\partial \dot{x}_{n}}\right)$. Similarly the partial derivatives of the vector function $g$ and the vector function $h$ can be written, using matrices with $q$ rows and $s$ rows instead of one, respectively.

Let $S$ denote the set of all feasible points of (MVPP), i.e.:

$$
S=\{x \in X \text { verifying the constraints of (MVPP) }\} .
$$

For notational convenience, we use $\varphi(t, x, \dot{x})$ for $\varphi(t, x(t), \dot{x}(t)), x$ for $x(t)$ and $\dot{x}$ for $\dot{x}(t)$.

Definition 1 A feasible solution $\bar{x}$ in the considered multiobjective variational control problem (MVPP) is said to be efficient of (MVPP) if there exists no another $x \in S$ such that

$$
\int_{a}^{b} f(t, x, \dot{x}) d t \leq \int_{a}^{b} f(t, \bar{x}, \bar{x}) d t
$$

that is, there exists no another $x \in S$ such that

$$
\begin{aligned}
& \int_{a}^{b} f^{i}(t, x, \dot{x}) d t \leqq \int_{a}^{b} f^{i}(t, \bar{x}, \bar{x}) d t, \quad \forall i \in A, \\
& \int_{a}^{b} f^{r}(t, x, \dot{x}) d t<\int_{a}^{b} f^{r}(t, \bar{x}, \bar{x}) d t \quad \text { for some } r \in A .
\end{aligned}
$$

Now, we give a definition of convexity of a functional $\Phi: I \times R^{n} \times R^{n} \times R^{n} \times$ $R^{n} \times R^{n} \times R \rightarrow R$.

Definition 2 Let $\Phi: I \times R^{n} \times R^{n} \times R^{n} \times R^{n} \times R^{n} \times R \rightarrow R$. A functional $\Phi(t, x, \dot{x}, \bar{x}, \dot{x} ; \cdot)$ is convex on $R^{n+1}$ if, for any $x, \bar{x}, \dot{x}, \dot{x} \in R^{n}$, the inequality

$$
\begin{aligned}
& \Phi\left(t, x, \dot{x}, \bar{x}, \bar{x} ;\left(\lambda\left(\xi_{1}, \rho_{1}\right)+(1-\lambda)\left(\xi_{2}, \rho_{2}\right)\right)\right) \\
& \quad \leqq \lambda \Phi\left(t, x, \dot{x}, \bar{x}, \bar{x}, \dot{x} ;\left(\xi_{1}, \rho_{1}\right)\right)+(1-\lambda) \Phi\left(t, x, \dot{x}, \bar{x}, \dot{x} ;\left(\xi_{2}, \rho_{2}\right)\right)
\end{aligned}
$$

holds for any $\xi_{1}, \xi_{2} \in R^{n}, \rho_{1}, \rho_{2} \in R$ and for all $\lambda \in[0,1]$. 
Let $\Psi: X \rightarrow R$ be defined by $\Psi(x)=\int_{a}^{b} \varphi(t, x, \dot{x}) d t$, where $\varphi: I \times X \times X \rightarrow R$ and, for notational convenience, we use $\varphi(t, x, \dot{x})$ for $\varphi(t, x(t), \dot{x}(t))$. The following definitions introduce the concepts of $(\Phi, \rho)$-invexity and of $(\Phi, \rho)$-incavity for the functional $\Psi$.

Definition 3 If there exist a real number $\rho$ and a functional $\Phi: I \times R^{n} \times R^{n} \times R^{n} \times R^{n} \times$ $R^{n} \times R \rightarrow R$, where $\Phi(t, x, \dot{x}, \bar{x}, \bar{x} ; \cdot)$ is convex on $R^{n+1}, \Phi(t, x, \dot{x}, \bar{x}, \bar{x} ;(o, a)) \geqq 0$ for every $x \in X$ and any $a \in R_{+}$such that the relation

$$
\Psi(x)-\Psi(\bar{x}) \geqq \int_{a}^{b} \Phi\left(t, x, \dot{x}, \bar{x}, \dot{x} ;\left(\varphi_{x}(t, \bar{x}, \dot{\bar{x}})-\frac{d}{d t} \varphi_{\dot{x}}(t, \bar{x}, \dot{\bar{x}}), \rho\right)\right) d t(>)
$$

holds for all $x \in X,(x \neq \bar{x})$, then the functional $\Psi$ is said to be (strictly) $(\Phi, \rho)$-invex at $\bar{x} \in X$ on $X$.

If (1) is satisfied for every $\bar{x} \in X$, then $\Psi$ is said to be (strictly) ( $\Phi, \rho$ )-invex on $X$.

Definition 4 If there exist a real number $\rho$ and a functional $\Phi: I \times R^{n} \times R^{n} \times R^{n} \times R^{n} \times$ $R^{n} \times R \rightarrow R$, where $\Phi(t, x, \dot{x}, \bar{x}, \bar{x}, \cdot)$ is convex on $R^{n+1}, \Phi(t, x, \dot{x}, \bar{x}, \bar{x} ;(o, a)) \geqq 0$ for every $x \in X$ and any $a \in R_{+}$such that the relation

$$
\Psi(x)-\Psi(\bar{x}) \leqq \int_{a}^{b} \Phi\left(t, x, \dot{x}, \bar{x}, \dot{\bar{x}} ;\left(\varphi_{x}(t, \bar{x}, \dot{\bar{x}})-\frac{d}{d t} \varphi_{\dot{x}}(t, \bar{x}, \dot{\bar{x}}), \rho\right)\right) d t(<)
$$

holds for all $x \in X,(x \neq \bar{x})$, then the functional $\Psi$ is said to be (strictly) $(\Phi, \rho)$ incave at $\bar{x} \in X$ on $X$. If (2) is satisfied for every $\bar{x} \in X$, then $\Psi$ is said to be (strictly) $(\Phi, \rho)$-incave on $X$.

Now, we give an example of a multiobjective variational control problem in which the functions involved are $(\Phi, \rho)$-invex.

Example 5 Let $n=2$ and consider the following multiobjective variational control problem:

$$
\begin{aligned}
& \text { Minimize } \int_{a}^{b} f(t, x(t), \dot{x}(t)) d t \\
&=\left(\int_{a}^{b}\left(x_{1}^{2}(t)+x_{2}^{2}(t)\right) d t, \int_{a}^{b}\left(x_{1}^{2}(t)+x_{1}(t) x_{2}(t)+x_{2}^{2}(t)\right) d t\right)
\end{aligned}
$$

subject to $g(t, x(t), \dot{x}(t))=x_{1}(t) x_{2}(t)-1 \leqq 0, t \in I$. 
Now, we show that the functions constituting the considered multiobjective variational control problem (MVPP1) are $(\Phi, \rho)$-invex. Indeed, we set

$$
\begin{gathered}
\Phi_{1}\left(t, x, \dot{x}, \bar{x}, \dot{\bar{x}} ;\left(\varphi_{x}(t, \bar{x}, \dot{\bar{x}})-\frac{d}{d t} \varphi_{\dot{x}}(t, \bar{x}, \dot{\bar{x}}), \rho_{1}\right)\right) \\
=-\bar{x}_{1} \varphi_{x_{1}}(t, \bar{x}, \dot{\bar{x}})-\bar{x}_{2} \varphi_{x_{2}}(t, \bar{x}, \dot{\bar{x}})+2\left(2^{\rho_{1}}-1\right)\left(\left|x_{1} x_{2}\right|+\left|\bar{x}_{1} \bar{x}_{2}\right|\right), \\
\rho_{f_{1}}=0, \quad \rho_{f_{2}}=0, \quad \rho_{g}=-1,
\end{gathered}
$$

where $\varphi$ is equal to $f_{1}, f_{2}$ or $g$, respectively, $\rho_{1}$ is equal to $\rho_{f_{1}}, \rho_{f_{2}}$ or $\rho_{g}$, respectively. Then, by Definition 3 , it can be proved that all functions constituting the considered multiobjective variational control problem (MVPP1) are $(\Phi, \rho)$-invex with respect to $\Phi_{1}$ and $\rho_{1}$ defined above.

Remark 6 Note that, in general, the functions constituting optimization problem are $(\Phi, \rho)$-invex with respect to more than one $\Phi$ and $\rho$. Indeed, it is not difficult to show that, in the case of the considered multiobjective variational control problem (MVPP1), the functions constituting it are $(\Phi, \rho)$-invex also with respect to $\Phi_{2}$ and $\rho_{2}$ defined as follows:

$$
\begin{aligned}
& \Phi_{2}\left(t, x, \dot{x}, \bar{x}, \dot{\bar{x}} ;\left(\varphi_{x}(t, \bar{x}, \dot{\bar{x}})-\frac{d}{d t} \varphi_{\dot{x}}(t, \bar{x}, \dot{\bar{x}}), \rho_{2}\right)\right) \\
& \quad=-\bar{x}_{1} \varphi_{x_{1}}(t, \bar{x}, \dot{\bar{x}})-\bar{x}_{2} \varphi_{x_{2}}(t, \bar{x}, \dot{\bar{x}})+2\left(2^{\rho_{2}}-1\right)\left|x_{1} x_{2}+\bar{x}_{1} \bar{x}_{2}\right|, \\
& \rho_{f_{1}}=1, \quad \rho_{f_{2}}=1, \quad \rho_{g}=-1 .
\end{aligned}
$$

Remark 7 Note that not all functions constituting the considered multiobjective variational control problem (MVPP1) are invex (see [14]). Indeed, the constraint $g$ is not invex with respect to any function $\eta: I \times R^{2} \times R^{2} \times R^{2} \times R^{2} \rightarrow R^{2}$, since a stationary point is not its global minimizer (see [5]). Therefore, it is not possible to prove the duality results established in the paper under invexity assumptions imposed on the functions constituting the considered multiobjective variational control problem (MVPP1). Further, since most of the generalized convex functions possess the fundamental property invexity mentioned above, therefore, they can not be used to prove duality result for such nonconvex multiobjective variational control problems as problem (MVPP1). However, we are in a position to prove various duality results for such nonconvex multiobjective variational control problems under $(\Phi, \rho)$-invexity. In other words, the duality results established in this paper under $(\Phi, \rho)$-invexity are true also for such nonconvex multiobjective variational control problems as problem (MVPP1) considered in Example 5 for which most of the generalized convexity notions may avoid.

\section{Mond-Weir type vector duality}

In this section, we prove duality results between the considered multiobjective variational control problem (MVPP) and its Mond-Weir type vector variational control dual problem (VMWD) defined as follows 


$$
\begin{gathered}
\text { Maximize } \int_{a}^{b} f(t, y(t), \dot{y}(t)) d t \\
\text { subject to }\left[\lambda^{T} f_{y}(t, y(t), \dot{y}(t))+\xi(t)^{T} g_{y}(t, y(t), \dot{y}(t))+\zeta(t)^{T} h_{y}(t, y(t), \dot{y}(t))\right] \\
=\frac{d}{d t}\left[\lambda^{T} f_{\dot{y}}(t, y(t), \dot{y}(t))+\xi(t)^{T} g_{\dot{y}}(t, y(t), \dot{y}(t))+\zeta(t)^{T} h_{\dot{y}}(t, y(t), \dot{y}(t))\right], \quad t \in I, \\
\int_{a}^{b} \xi(t)^{T} g(t, y(t), \dot{y}(t)) d t \geqq 0, \\
\int_{a}^{b} \zeta(t)^{T} h(t, y(t), \dot{y}(t)) d t=0, \\
y(a)=\alpha, \quad y(b)=\beta, \\
\lambda \geq 0, \quad \lambda^{T} e=1, \quad \xi(t) \geqq 0,
\end{gathered}
$$

where $e=(1, \ldots, 1) \in R^{p}$ is a $p$-dimensional vector. It may be noted here that the above dual constraints are written using the Karush-Kuhn-Tucker necessary optimality conditions for the considered multiobjective programming problem (MVPP) (see Theorem 9). For notational convenience, we use $\xi$ for $\xi(t)$ and $\zeta$ for $\zeta(t)$.

Let $\Omega_{M W}$ be the set of all feasible solutions $(y, \lambda, \xi, \zeta)$ in Mond-Weir type dual problem (VMWD). We denote by $Y$ the set $Y=\left\{y \in X:(y, \lambda, \xi, \zeta) \in \Omega_{M W}\right\}$.

Theorem 8 (Weak duality): Let $x$ and $(y, \lambda, \xi, \zeta)$ be any feasible solutions in multiobjective variational control problems (MVPP) and (VMWD), respectively. Further, assume that the following hypotheses are fulfilled:

(a) $\int_{a}^{b} f^{i}(t, \cdot, \cdot) d t, i=1, \ldots, k$, is strictly $\left(\Phi, \rho_{f_{i}}\right)$-invex at y on $S \cup Y$,

(b) $\int_{a}^{b} g^{j}(t, \cdot, \cdot) d t, j=1, \ldots, q$, is $\left(\Phi, \rho_{g_{j}}\right)$-invex at $y$ on $S \cup Y$,

(c) $\int_{a}^{b} h^{k}(t, \cdot, \cdot) d t, k \in K^{+}(t)=\left\{k \in K: \zeta_{k}(t)>0\right\}$, is ( $\left.\Phi, \rho_{h_{k}}\right)$-invex at y on $S \cup Y$,

(d) $\int_{a}^{b}\left(-h^{k}(t, \cdot, \cdot)\right) d t, k \in K^{-}(t)=\left\{k \in K: \zeta_{k}(t)<0\right\}$, is $\left(\Phi, \rho_{h_{k}}\right)$-invex at y on $S \cup Y$

(e) $\sum_{i=1}^{p} \lambda_{i} \rho_{f_{i}}+\sum_{j=1}^{q} \xi_{j} \rho_{g_{j}}+\sum_{k \in K^{+}(t)} \zeta_{k} \rho_{h_{k}}-\sum_{k \in K^{-}(t)} \zeta_{k} \rho_{h_{k}} \geqq 0$.

Then, the following cannot hold

$$
\int_{a}^{b} f(t, x, \dot{x}) d t \leq \int_{a}^{b} f(t, y, \dot{y}) d t
$$

that is, the following cannot hold

$$
\int_{a}^{b} f^{i}(t, x, \dot{x}) d t \leqq \int_{a}^{b} f^{i}(t, y, \dot{y}) d t, \quad \forall i \in A,
$$


and

$$
\int_{a}^{b} f^{r}(t, x, \dot{x}) d t<\int_{a}^{b} f^{r}(t, y, \dot{y}) d t \text { for some } r \in A \text {. }
$$

Proof We proceed by contradiction. Suppose, contrary to the result, that the inequalities (3) and (4) are satisfied. Since the hypotheses (a)-(d) are fulfilled, therefore, by Definitions 3 and 4, the following inequalities

$$
\begin{aligned}
& \int_{a}^{b} f^{i}(t, x, \dot{x}) d t-\int_{a}^{b} f^{i}(t, y, \dot{y}) d t \\
& >\int_{a}^{b} \Phi\left(t, x, \dot{x}, y, \dot{y} ;\left(f_{y}^{i}(t, y, \dot{y})-\frac{d}{d t} f_{\dot{y}}^{i}(t, y, \dot{y}), \rho_{f_{i}}\right)\right) d t, \quad i \in A \\
& \int_{a}^{b} g^{j}(t, x, \dot{x}) d t-\int_{a}^{b} g^{j}(t, y, \dot{y}) d t \\
& \geqq \int_{a}^{b} \Phi\left(t, x, \dot{x}, y, \dot{y} ;\left(g_{y}^{j}(t, y, \dot{y})-\frac{d}{d t} g_{\dot{y}}^{j}(t, y, \dot{y}), \rho_{g_{j}}\right)\right) d t, j \in J \\
& \int_{a}^{b} h^{k}(t, x, \dot{x}) d t-\int_{a}^{b} h^{k}(t, y, \dot{y}) d t \\
& \geqq \int_{a}^{b} \Phi\left(t, x, \dot{x}, y, \dot{y} ;\left(h_{y}^{k}(t, y, \dot{y})-\frac{d}{d t} h_{\dot{y}}^{k}(t, y, \dot{y}), \rho_{h_{k}}\right)\right) d t, k \in K^{+}(t) \\
& \quad \int_{a}^{b} \Phi\left(t, x, \dot{x}, y, \dot{y} ;\left(-h_{y}^{k}(t, y, \dot{y})-\frac{d}{d t}\left[-h_{\dot{y}}^{k}(t, y, \dot{y})\right], \rho_{h_{k}}\right)\right) d t \quad k \in K^{-}(t) \\
& \quad-\int_{a}^{b} h^{k}(t, x, \dot{x}) d t+\int_{a}^{b} h^{k}(t, y, \dot{y}) d t
\end{aligned}
$$

holds. Combining (3)-(5) and taking into account that $\lambda \geq 0$, we get

$$
\int_{a}^{b} \lambda_{i} \Phi\left(t, x, \dot{x}, y, \dot{y} ;\left(f_{y}^{i}(t, y, \dot{y})-\frac{d}{d t} f_{y}^{i}(t, y, \dot{y}), \rho_{f_{i}}\right)\right) d t \leqq 0, \quad i \in A
$$


and

$$
\int_{a}^{b} \lambda_{r} \Phi\left(t, x, \dot{x}, y, \dot{y} ;\left(f_{y}^{r}(t, y, \dot{y})-\frac{d}{d t} f_{y}^{r}(t, y, \dot{y}), \rho_{f_{i}}\right)\right) d t<0
$$

for at least one $r \in A$.

Since $(y, \lambda, \xi, \zeta) \in \Omega_{M W}$, adding both sides of the inequalities (6)-(8), we obtain

$$
\begin{aligned}
& \int_{a}^{b} \sum_{j=1}^{q} \xi_{j} g^{j}(t, x, \dot{x}) d t-\int_{a}^{b} \sum_{j=1}^{q} \xi_{j} g^{j}(t, y, \dot{y}) d t \\
& \geqq \int_{a} \sum_{j=1}^{q} \xi_{j} \Phi\left(t, x, \dot{x}, y, \dot{y} ;\left(g_{y}^{j}(t, y, \dot{y})-\frac{d}{d t} g_{\dot{y}}^{j}(t, y, \dot{y}), \rho_{g_{j}}\right)\right) d t, \\
& \int_{a}^{b} \sum_{k \in K^{+}(t) \cup K^{-}(t)} \zeta_{k} h^{k}(t, x, \dot{x}) d t-\int_{a}^{b} \sum_{k \in K^{+}(t) \cup K^{-}(t)} \zeta_{k} h^{k}(t, y, \dot{y}) d t \\
& \geqq \int_{a}^{b} \sum_{k \in K^{+}(t)} \zeta_{k} \Phi\left(t, x, \dot{x}, y, \dot{y} ;\left(h_{y}^{k}(t, y, \dot{y})-\frac{d}{d t}\left[h_{\dot{y}}^{k}(t, y, \dot{y})\right], \rho_{h_{k}}\right)\right) d t \\
& +\int_{a}^{b} \sum_{k \in K^{-}(t)}\left(-\zeta_{k}\right) \Phi\left(t, x, \dot{x}, y, \dot{y} ;\left(-h_{y}^{k}(t, y, \dot{y})-\frac{d}{d t}\left[-h_{\dot{y}}^{k}(t, y, \dot{y})\right], \rho_{h_{k}}\right)\right) d t .
\end{aligned}
$$

Using the feasibility of $x$ and of $(y, \lambda, \xi, \zeta)$ in problems (MVPP) and (VMWD), respectively, the inequalities (11) and (12) yield, respectively,

$$
\begin{aligned}
& \int_{a}^{b} \sum_{j=1}^{q} \xi_{j} \Phi\left(t, x, \dot{x}, y, \dot{y} ;\left(g_{y}^{j}(t, y, \dot{y})-\frac{d}{d t}\left[g_{\dot{y}}^{j}(t, y, \dot{y})\right], \rho_{g_{j}}\right)\right) d t \leqq 0 \\
& \int_{a}^{b} \sum_{k \in K^{+}(t)} \zeta_{k} \Phi\left(t, x, \dot{x}, y, \dot{y} ;\left(h_{y}^{k}(t, y, \dot{y})-\frac{d}{d t}\left[h_{\dot{y}}^{k}(t, y, \dot{y})\right], \rho_{h_{k}}\right)\right) \\
& +\int_{a}^{b} \sum_{k \in K^{-}(t)}\left(-\zeta_{k}\right) \Phi\left(t, x, \dot{x}, y, \dot{y} ;\left(-h_{y}^{k}(t, y, \dot{y})-\frac{d}{d t}\left[-h_{y}^{k}(t, y, \dot{y})\right], \rho_{h_{k}}\right)\right) d t \leqq 0 .
\end{aligned}
$$


Combining (9), (10), (13) and (14), we get

$$
\begin{aligned}
& \int_{a}^{b} \sum_{i=1}^{p} \lambda_{i} \Phi\left(t, x, \dot{x}, y, \dot{y} ;\left(f_{y}^{i}(t, y, \dot{y})-\frac{d}{d t} f_{\dot{y}}^{i}(t, y, \dot{y}), \rho_{f_{i}}\right)\right) d t \\
& +\int_{a} \sum_{j=1}^{q} \xi_{j} \Phi\left(t, x, \dot{x}, y, \dot{y} ;\left(g_{y}^{j}(t, y, \dot{y})-\frac{d}{d t} g_{\dot{y}}^{j}(t, y, \dot{y}), \rho_{g_{j}}\right)\right) d t \\
& +\int_{a}^{b} \sum_{k \in K^{+}(t)} \zeta_{k} \Phi\left(t, x, \dot{x}, y, \dot{y} ;\left(h_{y}^{k}(t, y, \dot{y})-\frac{d}{d t} h_{\dot{y}}^{j}(t, y, \dot{y}), \rho_{h_{k}}\right)\right) d t \\
& +\int_{a}^{b} \sum_{k \in K^{-}(t)}\left(-\zeta_{k}\right) \Phi\left(t, x, \dot{x}, y, \dot{y} ;\left(-h_{y}^{k}(t, y, \dot{y})-\frac{d}{d t}\left[-h_{y}^{k}(t, y, \dot{y})\right], \rho_{h_{k}}\right)\right) d t<0 .
\end{aligned}
$$

We denote

$$
\begin{gathered}
\tilde{\lambda}_{i}=\frac{\lambda_{i}}{\sum_{i=1}^{p} \lambda_{i}+\sum_{j=1}^{q} \xi_{j}(t)+\sum_{k \in K^{+}(t)} \zeta_{k}(t)-\sum_{k \in K^{-}(t)} \zeta_{k}(t)}, \quad i \in A, \quad(16) \\
\widetilde{\xi}_{j}(t)=\frac{\xi_{j}(t)}{\sum_{i=1}^{p} \lambda_{i}+\sum_{j=1}^{q} \xi_{j}(t)+\sum_{k \in K^{+}(t)} \zeta_{k}(t)-\sum_{k \in K^{-}(t)} \zeta_{k}(t)}, \quad j \in J, \quad(17) \\
\widetilde{\zeta}_{k}(t)=\frac{\zeta_{k}(t)}{\sum_{i=1}^{p} \lambda_{i}+\sum_{j=1}^{q} \xi_{j}(t)+\sum_{k \in K^{+}(t)} \zeta_{k}(t)-\sum_{k \in K^{-}(t)} \zeta_{k}(t)}, \quad k \in K^{+}(t), \\
\widetilde{\zeta}_{k}(t)=\frac{-\zeta_{k}(t)}{\sum_{i=1}^{p} \lambda_{i}+\sum_{j=1}^{q} \xi_{j}(t)+\sum_{k \in K^{+}(t)} \zeta_{k}(t)-\sum_{k \in K^{-}(t)} \zeta_{k}(t)},
\end{gathered}
$$

By (16)-(19), it follows that $0 \leqq \widetilde{\lambda}_{i} \leqq 1, i \in A$, but $\tilde{\lambda}_{i}>0$ for at least one $i \in A$, $0 \leqq \widetilde{\xi}_{j}(t) \leqq 1, j \in J, 0 \leqq \widetilde{\zeta}_{k}(t) \leqq 1, k \in K$, and, moreover,

$$
\sum_{i=1}^{p} \widetilde{\lambda}_{i}+\sum_{j=1}^{q} \widetilde{\xi}_{j}(t)+\sum_{k \in K^{+}(t)} \widetilde{\zeta}_{k}(t)+\sum_{k \in K^{-}(t)} \widetilde{\zeta}_{k}(t)=1
$$

Combining (15)-(19), we get

$$
\int_{a}^{b} \sum_{i=1}^{p} \widetilde{\lambda}_{i} \Phi\left(t, x, \dot{x}, y, \dot{y} ;\left(f_{y}^{i}(t, y, \dot{y})-\frac{d}{d t} f_{\dot{y}}^{i}(t, y, \dot{y}), \rho_{f_{i}}\right)\right) d t
$$




$$
\begin{aligned}
& +\int_{a}^{b} \sum_{j=1}^{q} \widetilde{\xi}_{j} \Phi\left(t, x, \dot{x}, y, \dot{y} ;\left(g_{y}^{j}(t, y, \dot{y})-\frac{d}{d t} g_{y}^{j}(t, y, \dot{y}), \rho_{g_{j}}\right)\right) d t \\
& +\int_{a}^{b} \sum_{k \in K^{+}(t)} \widetilde{\zeta}_{k} \Phi\left(t, x, \dot{x}, y, \dot{y} ;\left(h_{y}^{k}(t, y, \dot{y})-\frac{d}{d t} h_{y}^{k}(t, y, \dot{y}), \rho_{h_{k}}\right)\right) d t \\
& +\int_{a}^{b} \sum_{k \in K^{-}(t)} \widetilde{\zeta}_{k} \Phi\left(t, x, \dot{x}, y, \dot{y} ;\left(-h_{y}^{k}(t, y, \dot{y})-\frac{d}{d t}\left[-h_{y}^{k}(t, y, \dot{y})\right], \rho_{h_{k}}\right)\right) d t<0 .
\end{aligned}
$$

Then, by Definition 3, it follows that the functional $\Phi(t, x, \dot{x}, y, \dot{y}, \cdot)$ is convex on $R^{n+1}$. Thus, by (21) and (20), Definition 2 implies

$$
\begin{aligned}
& \int_{a}^{b} \Phi\left(t, x, \dot{x}, y, \dot{y} ;\left(\left[\sum_{i=1}^{p} \widetilde{\lambda}_{i} f_{y}^{i}(t, y, \dot{y})+\sum_{j=1}^{q} \widetilde{\xi}_{j} g_{y}^{j}(t, y, \dot{y})\right.\right.\right. \\
& \left.+\sum_{k \in K^{+}(t)} \widetilde{\zeta}_{k} h_{y}^{k}(t, y, \dot{y})+\sum_{k \in K^{-}(t)}\left(-\widetilde{\zeta}_{k}\right) h_{y}^{k}(t, y, \dot{y})\right] \\
& -\frac{d}{d t}\left[\sum_{i=1}^{p} \widetilde{\lambda}_{i} f_{\dot{y}}^{i}(t, y, \dot{y})+\sum_{j=1}^{q} \widetilde{\xi}_{j} g_{\dot{y}}^{j}(t, y, \dot{y})+\sum_{k \in K^{+}(t)} \widetilde{\zeta}_{k} h_{\dot{y}}^{k}(t, y, \dot{y})\right. \\
& \left.\left.\left.+\sum_{k \in K^{-}(t)}\left(-\widetilde{\zeta}_{k}\right) h_{\dot{y}}^{k}(t, y, \dot{y})\right], \sum_{i=1}^{p} \widetilde{\lambda}_{f_{i}}+\sum_{j=1}^{q} \widetilde{\xi}_{j} \rho_{g_{j}}+\sum_{k \in K^{+}(t) \cup K^{-}(t)} \widetilde{\zeta}_{k} \rho_{h_{k}}\right)\right) d t<0 .
\end{aligned}
$$

Hence, the first constraint of (VMWD) yields

$$
\int_{a}^{b} \Phi\left(t, x, \dot{x}, y, \dot{y} ;\left(0, \sum_{i=1}^{p} \widetilde{\lambda}_{i} \rho_{f_{i}}+\sum_{j=1}^{q} \widetilde{\xi}_{j} \rho_{g_{j}}+\sum_{k \in K^{+}(t) \cup K^{-}(t)} \widetilde{\zeta}_{k} \rho_{h_{k}}\right)\right) d t<0 .
$$

From the hypothesis e), it follows that

$$
\sum_{i=1}^{p} \widetilde{\lambda}_{i} \rho_{f_{i}}+\sum_{j=1}^{q} \widetilde{\xi}_{j}(t) \rho_{g_{j}}+\sum_{k=1}^{s} \widetilde{\zeta}_{k}(t) \rho_{h_{k}} \geqq 0
$$


By Definition 3, it follows that $\Phi(t, x, \dot{x}, y, \dot{y} ;(0, a)) \geqq 0$ for any $a \in R_{+}$. Thus, (23) implies that the following inequality

$$
\int_{a}^{b} \Phi\left(t, x, \dot{x}, y, \dot{y} ; \quad\left(0, \sum_{i=1}^{p} \widetilde{\lambda}_{i} \rho_{f_{i}}+\sum_{j=1}^{q} \widetilde{\xi}_{j} \rho_{g_{j}}+\sum_{k \in K^{+}(t) \cup K^{-}(t)} \widetilde{\zeta}_{k} \rho_{h_{k}}\right)\right) d t \geqq 0
$$

holds, contradicting (22).

In order to formulate strong duality theorem, we give the Karush-Kuhn-Tucker necessary optimality conditions for the considered multiobjective variational control problem (MVPP). This theorem is the continuous version of Theorem 2.2 [22] (see also $[2,4,12,13])$.

Theorem 9 Let $\bar{x}$ be a normal efficient solution in problem (MVPP) at which the Kuhn-Tucker constraint qualification is satisfied. Then, there exist $\bar{\lambda} \in R^{p}$ and the piecewise smooth functions $\bar{\xi}(\cdot): I \rightarrow R^{m}$ and $\bar{\zeta}(\cdot): I \rightarrow R^{s}$ such that

$$
\begin{aligned}
& \bar{\lambda}^{T} f_{x}(t, \bar{x}, \dot{\bar{x}})+\bar{\xi}^{T} g_{x}(t, \bar{x}, \dot{\bar{x}})+\bar{\zeta}^{T} h_{x}(t, \bar{x}, \dot{\bar{x}}) \\
& \quad=\frac{d}{d t}\left[\bar{\lambda}^{T} f_{\dot{x}}(t, \bar{x}, \dot{\bar{x}})+\bar{\xi}^{T} g_{\dot{x}}(t, \bar{x}, \dot{\bar{x}})+\bar{\zeta}^{T} h_{\dot{x}}(t, \bar{x}, \overline{\bar{x}})\right], \quad t \in I, \\
& \int_{a}^{b} \bar{\xi}^{T} g(t, \bar{x}, \dot{\bar{x}}) d t=0, \\
& \bar{\lambda} \geq 0, \quad \bar{\lambda}^{T} e=1, \quad \bar{\xi}(t) \geqq 0 .
\end{aligned}
$$

Theorem 10 (Strong duality): Let $\bar{x}$ be an efficient solution in the considered multiobjective variational control problem (MVPP). Further, assume that the Kuhn-Tucker constraint qualification is satisfied for $(M V P P)$. Then, there exist $\bar{\lambda} \in R_{+}^{p}$ and the piecewise smooth functions $\bar{\xi}(\cdot): I \rightarrow R^{m}$ and $\bar{\zeta}(\cdot): I \rightarrow R^{s}$ such that $(\bar{x}, \bar{\lambda}, \bar{\xi}(t), \bar{\zeta}(t))$ is a feasible solution for problem (VMWD). If also the weak duality theorem (Theorem 8 ) holds between (MVPP) and (VMWD), then $(\bar{x}, \bar{\lambda}, \bar{\xi}(t), \bar{\zeta}(t))$ is an efficient solution in Mond-Weir type dual problem (VMWD) and the objective functions values are equal.

Proof By assumption, $\bar{x}$ is an efficient solution in the considered multiobjective variational control problem (MVPP). Hence, by Theorem 9, there exist $\bar{\lambda} \in R^{p}$ and the piecewise smooth functions $\bar{\xi}(\cdot): I \rightarrow R^{m}$ and $\bar{\zeta}(\cdot): I \rightarrow R^{s}$ such that the KarushKuhn-Tucker optimality conditions (24)-(26) are satisfied. Thus, $(\bar{x}, \bar{\lambda}, \bar{\xi}(t), \bar{\zeta}(t))$ is a feasible solution in Mond-Weir dual problem (VMWD) and the two objective functionals have the same values. Efficiency of $(\bar{x}, \bar{\lambda}, \bar{\xi}(t), \bar{\zeta}(t))$ in problem (VMWD) follows directly from weak duality theorem (Theorem 8 ).

Proposition 11 Let $(\bar{x}, \bar{\lambda}, \bar{\xi}(t), \bar{\zeta}(t))$ be a feasible solution in Mond-Weir type dual problem (VMWD) such that $\bar{x} \in S$. Further, assume that the following hypotheses are fulfilled: 
(a) $\int_{a}^{b} f^{i}(t, \cdot, \cdot) d t, i=1, \ldots, k$, is strictly $\left(\Phi, \rho_{f_{i}}\right)$-invex at $\bar{x}$ on $S$,

(b) $\int_{a}^{b} g^{j}(t, \cdot, \cdot) d t, j=1, \ldots, q$, is $\left(\Phi, \rho_{g_{j}}\right)$-invex at $\bar{x}$ on $S$,

(c) $\int_{a}^{b} h^{k}(t, \cdot, \cdot) d t, k \in K^{+}(t)=\left\{k \in K: \bar{\zeta}_{k}(t)>0\right\}$, is ( $\left.\Phi, \rho_{h_{k}}\right)$-invex at $\bar{x}$ on $S$,

(d) $\int_{a}^{b}\left(-h^{k}(t, \cdot, \cdot)\right) d t, k \in K^{-}(t)=\left\{k \in K: \bar{\zeta}_{k}(t)<0\right\}$, is $\left(\Phi, \rho_{h_{k}}\right)$-invex at $\bar{x}$ on $S$,

(e) $\sum_{i=1}^{p} \bar{\lambda}_{i} \rho_{f_{i}}+\sum_{j=1}^{q} \bar{\xi}_{j} \rho_{g_{j}}+\sum_{k \in K^{+}(t)} \bar{\zeta}_{k} \rho_{h_{k}}-\sum_{k \in K^{-}(t)} \bar{\zeta}_{k} \rho_{h_{k}} \geqq 0$.

Then $\bar{x}$ is efficient in the considered multiobjective variational control problem (MVPP).

Proof Proof follows from the weak duality theorem (Theorem 8).

The following result follows directly from the above proposition.

Theorem 12 (Converse duality): Let $(\bar{x}, \bar{\lambda}, \bar{\xi}(t), \bar{\zeta}(t))$ be an efficient solution in Mond-Weir type vector variational control dual problem (VMWD) and $\bar{x} \in S$. Further, assume that the hypotheses (a)-(e) of Proposition 11 are fulfilled. Then $\bar{x}$ is efficient in the considered multiobjective variational control problem (MVPP).

Theorem 13 (Strict converse duality): Let $\bar{x}$ and $(\bar{y}, \bar{\lambda}, \bar{\xi}(t), \bar{\zeta}(t))$ be any feasible solutions in problems (MVPP) and (VMWD), respectively, such that

$$
\int_{a}^{b} \sum_{i=1}^{p} \bar{\lambda}_{i} f^{i}(t, \bar{x}, \dot{\bar{x}}) d t \leqq \int_{a}^{b} \sum_{i=1}^{p} \bar{\lambda}_{i} f^{i}(t, \bar{y}, \dot{\bar{y}}) d t .
$$

Further, assume that the following hypotheses are fulfilled:

(a) $\int_{a}^{b} f^{i}(t, \cdot, \cdot) d t, i=1, \ldots, k$, is strictly $\left(\Phi, \rho_{f_{i}}\right)$-invex at $\bar{y}$ on $S \cup Y$,

(b) $\int_{a}^{b} g^{j}(t, \cdot, \cdot) d t, j=1, \ldots, q$, is $\left(\Phi, \rho_{g_{j}}\right)$-invex at $\bar{y}$ on $S \cup Y$,

(c) $\int_{a}^{b} h^{k}(t, \cdot, \cdot) d t, k \in K^{+}(t)=\left\{k \in K: \bar{\zeta}_{k}(t)>0\right\}$, is $\left(\Phi, \rho_{h_{k}}\right)$-invex at $\bar{y}$ on $S \cup Y$,

(d) $\int_{a}^{b}\left(-h^{k}(t, \cdot, \cdot)\right) d t, k \in K^{-}(t)=\left\{k \in K: \bar{\zeta}_{k}(t)<0\right\}$, is $\left(\Phi, \rho_{h_{k}}\right)$-invex at $\bar{y}$ on $S \cup Y$,

(e) $\sum_{i=1}^{p} \bar{\lambda}_{i} \rho_{f_{i}}+\sum_{j=1}^{q} \bar{\xi}_{j} \rho_{g_{j}}+\sum_{k \in K^{+}(t)} \bar{\zeta}_{k} \rho_{h_{k}}-\sum_{k \in K^{-}(t)} \bar{\zeta}_{k} \rho_{h_{k}} \geqq 0$.

Then $\bar{x}=\bar{y}$ and $\bar{y}$ is efficient in the considered multiobjective variational control problem (MVPP).

Proof Suppose, contrary to the result, that $\bar{x} \neq \bar{y}$. Since $\bar{x}$ and $(\bar{y}, \bar{\lambda}, \bar{\xi}(t), \bar{\zeta}(t))$ are any feasible solutions in problems (MVPP) and (VMWD), we have 


$$
\begin{aligned}
& \int_{a}^{b} \sum_{j=1}^{q} \bar{\xi}_{j} g^{j}(t, \bar{x}, \bar{x}) d t \leqq \int_{a}^{b} \sum_{j=1}^{q} \bar{\xi}_{j} g^{j}(t, \bar{y}, \bar{y}) d t, \\
& \int_{a}^{b} \sum_{k \in K} \bar{\zeta}_{k} h^{k}(t, \bar{x}, \dot{\bar{x}}) d t=\int_{a}^{b} \sum_{k \in K} \bar{\zeta}_{k} h^{k}(t, \bar{y}, \dot{y}) d t,
\end{aligned}
$$

By Definition 3, the hypotheses (b)-(d) yield

$$
\begin{aligned}
& \int_{a}^{b} g^{j}(t, \bar{x}, \dot{\bar{x}}) d t-\int_{a}^{b} g^{j}(t, \bar{y}, \dot{\bar{y}}) d t \\
& \geqq \int_{a}^{b} \Phi\left(t, \bar{x}, \dot{\bar{x}}, \bar{y}, \dot{\bar{y}} ;\left(g_{y}^{j}(t, \bar{y}, \dot{\bar{y}})-\frac{d}{d t}\left[g_{\dot{y}}^{j}(t, \bar{y}, \dot{\bar{y}})\right], \rho_{g_{j}}\right)\right) d t, \quad j \in J, \quad(30) \\
& \int_{a}^{b} h^{k}(t, \bar{x}, \dot{\bar{x}}) d t-\int_{a}^{b} h^{k}(t, \bar{y}, \dot{\bar{y}}) d t \\
& \geqq \int_{a}^{b} \Phi\left(t, \bar{x}, \dot{\bar{x}}, \bar{y}, \dot{\bar{y}} ;\left(h_{y}^{k}(t, \bar{y}, \dot{\bar{y}})-\frac{d}{d t}\left[h_{\dot{y}}^{k}(t, \bar{y}, \dot{\bar{y}})\right], \rho_{h_{k}}\right)\right) d t, \quad k \in K^{+}(t), \\
& -\int_{a}^{b} h^{k}(t, \bar{x}, \dot{\bar{x}}) d t+\int_{a}^{b} h^{k}(t, \bar{y}, \dot{\bar{y}}) d t \\
& \geqq \int_{a}^{b} \Phi\left(t, \bar{x}, \dot{\bar{x}}, \bar{y}, \dot{\bar{y}} ;\left(-h_{y}^{k}(t, \bar{y}, \dot{\bar{y}})-\frac{d}{d t}\left[-h_{\dot{y}}^{k}(t, \bar{y}, \dot{\bar{y}})\right], \rho_{h_{k}}\right)\right) d t, \quad k \in K^{-}(t) .
\end{aligned}
$$

After multiplying both sides of (30) by $\bar{\xi}_{j} \geqq 0, j \in J$, adding both sides of the obtained inequalities, by (28), we get

$$
\int_{a}^{b} \sum_{j=1}^{q} \bar{\xi}_{j} \Phi\left(t, \bar{x}, \bar{x}, \bar{y}, \dot{y} ;\left(g_{y}^{j}(t, \bar{y}, \dot{y})-\frac{d}{d t}\left[g_{\dot{y}}^{j}(t, \bar{y}, \dot{y})\right], \rho_{g_{j}}\right)\right) d t \leqq 0 .
$$

After multiplying both sides of (31) by $\bar{\zeta}_{k}>0, k \in K^{+}(t)$, and (32) by $-\bar{\zeta}_{k}, k \in$ $K^{-}(t)$, where $\bar{\zeta}_{k}<0, k \in K^{-}(t)$, adding both sides of the obtained inequalities, we get 


$$
\begin{aligned}
& \int_{a}^{b} \sum_{k \in K^{+}(t) \cup K^{-}(t)} \bar{\zeta}_{k} h^{k}(t, \bar{x}, \dot{\bar{x}}) d t-\int_{a}^{b} \sum_{k \in K^{+}(t) \cup K^{-}(t)} \bar{\zeta}_{k} h^{k}(t, \bar{y}, \dot{\bar{y}}) d t \\
& \geqq \int_{a}^{b} \sum_{k \in K^{+}(t)} \bar{\zeta}_{k} \Phi\left(t, \bar{x}, \dot{\bar{x}}, \bar{y}, \dot{\bar{y}} ;\left(h_{y}^{k}(t, \bar{y}, \dot{\bar{y}})-\frac{d}{d t}\left[h_{\dot{y}}^{k}(t, \bar{y}, \dot{\bar{y}})\right], \rho_{h_{k}}\right)\right) d t \\
& +\int_{a}^{b} \sum_{k \in K^{-}(t)}\left(-\bar{\zeta}_{k}\right) \Phi\left(t, \bar{x}, \dot{\bar{x}}, \bar{y}, \dot{\bar{y}} ;\left(-h_{y}^{k}(t, \bar{y}, \dot{\bar{y}})-\frac{d}{d t}\left[-h_{\dot{y}}^{k}(t, \bar{y}, \dot{y})\right], \rho_{h_{k}}\right)\right) d t .
\end{aligned}
$$

Thus, (29) and (34) give

$$
\begin{gathered}
\int_{a}^{b} \sum_{k \in K^{+}(t)} \bar{\zeta}_{k} \Phi\left(t, \bar{x}, \bar{x}, \bar{y}, \dot{y} ;\left(h_{y}^{k}(t, \bar{y}, \dot{y})-\frac{d}{d t}\left[h_{\dot{y}}^{k}(t, \bar{y}, \dot{y})\right], \rho_{h_{k}}\right)\right) d t \\
+\int_{a}^{b} \sum_{k \in K^{-}(t)}\left(-\bar{\zeta}_{k}\right) \Phi\left(t, \bar{x}, \dot{\bar{x}}, \bar{y}, \dot{\bar{y}} ;\left(-h_{y}^{k}(t, \bar{y}, \dot{\bar{y}})\right.\right. \\
\left.\left.-\frac{d}{d t}\left[-h_{\dot{y}}^{k}(t, \bar{y}, \dot{\bar{y}})\right], \rho_{h_{k}}\right)\right) d t \leqq 0 .
\end{gathered}
$$

We denote

$$
\tilde{\lambda}_{i}=\frac{\bar{\lambda}_{i}}{\sum_{i=1}^{p} \bar{\lambda}_{i}+\sum_{j=1}^{q} \bar{\xi}_{j}(t)+\sum_{k \in K^{+}(t)} \bar{\zeta}_{j}(t)-\sum_{k \in K^{-}(t)} \bar{\zeta}_{j}(t)}, \quad i \in A,
$$$$
\widetilde{\xi}_{j}(t)=\frac{\bar{\xi}_{j}(t)}{\sum_{i=1}^{p} \bar{\lambda}_{i}+\sum_{j=1}^{q} \bar{\xi}_{j}(t)+\sum_{k \in K^{+}(t)} \bar{\zeta}_{j}(t)-\sum_{k \in K^{-}(t)} \bar{\zeta}_{j}(t)}, \quad j \in J,
$$

$\widetilde{\zeta}_{k}(t)=\frac{\bar{\zeta}_{k}(t)}{\sum_{i=1}^{p} \bar{\lambda}_{i}+\sum_{j=1}^{q} \bar{\xi}_{j}(t)+\sum_{k \in K^{+}(t)} \bar{\zeta}_{j}(t)-\sum_{k \in K^{-}(t)} \bar{\zeta}_{j}(t)}, \quad k \in K^{+}(t)$,

$$
\widetilde{\zeta}_{k}(t)=\frac{-\bar{\zeta}_{k}(t)}{\sum_{i=1}^{p} \bar{\lambda}_{i}+\sum_{j=1}^{q} \bar{\xi}_{j}(t)+\sum_{k \in K^{+}(t)} \bar{\zeta}_{j}(t)-\sum_{k \in K^{-}(t)} \bar{\zeta}_{j}(t)}, \quad k \in K^{-}(t)
$$

By (36)-(39), it follows that $0 \leqq \widetilde{\lambda}_{i} \leqq 1, i \in A$, but $\widetilde{\lambda}_{i}>0$ for at least one $i \in A$, $0 \leqq \widetilde{\xi}_{j}(t) \leqq 1, j \in J, 0 \leqq \widetilde{\zeta}_{k}(t) \leqq 1, k \in K^{+}(t) \cup K^{-}(t)$, and, moreover, 


$$
\sum_{i=1}^{p} \widetilde{\lambda}_{i}+\sum_{j=1}^{q} \widetilde{\xi}_{j}(t)+\sum_{k \in K^{+}(t)} \widetilde{\zeta}_{k}(t)+\sum_{k \in K^{-}(t)} \widetilde{\zeta}_{k}(t)=1
$$

Then, hypothesis (e) implies

$$
\sum_{i=1}^{p} \widetilde{\lambda}_{i} \rho_{f_{i}}+\sum_{j=1}^{q} \widetilde{\xi}_{j}(t) \rho_{g_{j}}+\sum_{k \in K^{+}(t) \cup K^{-}(t)} \widetilde{\zeta}_{k}(t) \rho_{h_{k}} \geqq 0 .
$$

By Definition 3, it follows that $\Phi(t, \bar{x}, \bar{x}, \bar{y}, \bar{y} ;(0, a)) \geqq 0$ for any $a \in R_{+}$. Thus, (41) gives

$$
\int_{a}^{b} \Phi\left(t, \bar{x}, \dot{x}, \bar{y}, \dot{y} ;\left(0, \sum_{i=1}^{p} \widetilde{\lambda}_{i} \rho_{f_{i}}+\sum_{j=1}^{q} \widetilde{\xi}_{j}(t) \rho_{g_{j}}+\sum_{k \in K^{+}(t) \cup K^{-}(t)} \widetilde{\zeta}_{k}(t) \rho_{h_{k}}\right)\right) d t \geqq 0 .
$$

Thus, the first constraint of Mond-Weir type vector variational control dual problem (VMWD) yields

$$
\begin{aligned}
& \int_{a}^{b} \Phi\left(t, \bar{x}, \dot{\bar{x}}, \bar{y}, \dot{\bar{y}} ;\left(\sum_{i=1}^{p} \widetilde{\lambda}_{i} f_{y}^{i}(t, \bar{y}, \dot{\bar{y}})+\sum_{j=1}^{q} \widetilde{\xi}_{j} g_{y}^{j}(t, \bar{y}, \dot{\bar{y}})\right.\right. \\
& +\sum_{k \in K^{+}(t)} \widetilde{\zeta}_{k} h_{y}^{k}(t, \bar{y}, \bar{y})+\sum_{k \in K^{-}(t)}\left(-\widetilde{\zeta}_{k}\right) h_{y}^{k}(t, \bar{y}, \dot{\bar{y}}) \\
& -\frac{d}{d t}\left[\sum_{i=1}^{p} \widetilde{\lambda}_{i} f_{\dot{y}}^{i}(t, \bar{y}, \bar{y})+\sum_{j=1}^{q} \widetilde{\xi}_{j} g_{\dot{y}}^{j}(t, \bar{y}, \bar{y})\right. \\
& \left.+\sum_{k \in K^{+}(t)} \widetilde{\zeta}_{h_{y}}(t, \bar{y}, \bar{y})+\sum_{k \in K^{-}(t)}\left(-\widetilde{\zeta}_{k}\right) h_{\dot{y}}(t, \bar{y}, \dot{\bar{y}})\right], \\
& \left.\left.\sum_{i=1}^{p} \tilde{\lambda}_{i} \rho_{f_{i}}+\sum_{j=1}^{q} \widetilde{\xi}_{j} \rho_{g_{j}}+\sum_{k \in K^{+}(t) \cup K^{-}(t)} \widetilde{\zeta}_{k} \rho_{h_{k}}\right)\right) d t \geqq 0 .
\end{aligned}
$$

By Definition 3, it follows that the functional $\Phi(t, \bar{x}, \bar{x}, \bar{y}, \bar{y} ; \cdot)$ is convex on $R^{n+1}$. By (36)-(40), it follows that

$$
\begin{aligned}
& \int_{a}^{b} \sum_{i=1}^{p} \widetilde{\lambda}_{i} \Phi\left(t, \bar{x}, \dot{x}, \bar{y}, \dot{\bar{y}} ;\left(f_{y}^{i}(t, \bar{y}, \dot{\bar{y}})-\frac{d}{d t}\left[f_{\dot{y}}^{i}(t, \bar{y}, \dot{y})\right], \rho_{f_{i}}\right)\right) d t \\
& \quad+\int_{a}^{b} \sum_{j=1}^{q} \widetilde{\xi}_{j} \Phi\left(t, \bar{x}, \bar{x}, \bar{y}, \dot{y} ;\left(g_{y}^{j}(t, \bar{y}, \dot{y})-\frac{d}{d t}\left[g_{\dot{y}}^{j}(t, \bar{y}, \dot{\bar{y}})\right], \rho_{g_{j}}\right)\right) d t
\end{aligned}
$$




$$
\begin{aligned}
& +\int_{a}^{b} \sum_{k \in K^{+}(t)} \widetilde{\zeta}_{k} \Phi\left(t, \bar{x}, \dot{x}, \bar{y}, \dot{y} ;\left(h_{y}^{k}(t, \bar{y}, \dot{y})-\frac{d}{d t}\left[h_{y}^{k}(t, \bar{y}, \dot{y})\right], \rho_{h_{k}}\right)\right) d t \\
& +\int_{a}^{b} \sum_{k \in K^{-}(t)}\left(-\widetilde{\zeta}_{k}\right) \Phi\left(t, \bar{x}, \dot{\bar{x}}, \bar{y}, \dot{\bar{y}} ;\left(-h_{y}^{k}(t, \bar{y}, \dot{\bar{y}})-\frac{d}{d t}\left[-h_{y}^{k}(t, \bar{y}, \dot{y})\right], \rho_{h_{k}}\right)\right) d t \geqq 0 .
\end{aligned}
$$

Again by (36)-(40), it follows that

$$
\begin{aligned}
& \int_{a}^{b} \sum_{i=1}^{p} \bar{\lambda}_{i} \Phi\left(t, \bar{x}, \dot{\bar{x}}, \bar{y}, \dot{\bar{y}} ;\left(f_{y}^{i}(t, \bar{y}, \dot{\bar{y}})-\frac{d}{d t}\left[f_{\dot{y}}^{i}(t, \bar{y}, \dot{\bar{y}})\right], \rho_{f_{i}}\right)\right) d t \\
& +\int_{a}^{b} \sum_{j=1}^{q} \bar{\xi}_{j} \Phi\left(t, \bar{x}, \dot{\bar{x}}, \bar{y}, \dot{\bar{y}} ;\left(g_{y}^{j}(t, \bar{y}, \dot{\bar{y}})-\frac{d}{d t}\left[g_{\dot{y}}^{j}(t, \bar{y}, \dot{\bar{y}})\right], \rho_{g_{j}}\right)\right) d t \\
& +\int_{a}^{b} \sum_{k \in K^{+}(t)} \bar{\zeta}_{k} \Phi\left(t, \bar{x}, \dot{\bar{x}}, \bar{y}, \dot{\bar{y}} ;\left(h_{y}^{k}(t, \bar{y}, \dot{\bar{y}})-\frac{d}{d t}\left[h_{y}^{k}(t, \bar{y}, \dot{\bar{y}})\right], \rho_{h_{k}}\right)\right) d t \\
& +\int_{a}^{b} \sum_{k \in K^{-}(t)}\left(-\bar{\zeta}_{k}\right) \Phi\left(t, \bar{x}, \dot{\bar{x}}, \bar{y}, \bar{y} ;\left(-h_{y}^{k}(t, \bar{y}, \dot{\bar{y}})-\frac{d}{d t}\left[-h_{y}^{k}(t, \bar{y}, \bar{y})\right], \rho_{h_{k}}\right)\right) d t \geqq 0 .
\end{aligned}
$$

Combining (33), (35) and (45), we get

$$
\int_{a}^{b} \sum_{i=1}^{p} \bar{\lambda}_{i} \Phi\left(t, \bar{x}, \dot{\bar{x}}, \bar{y}, \dot{\bar{y}} ;\left(f_{y}^{i}(t, \bar{y}, \dot{\bar{y}})-\frac{d}{d t}\left[f_{\dot{y}}^{i}(t, \bar{y}, \bar{y})\right], \rho_{f_{i}}\right)\right) d t \geqq 0 .
$$

By hypothesis (a) and Definitions 3, it follows that

$$
\begin{aligned}
& \int_{a}^{b} f^{i}(t, \bar{x}, \dot{\bar{x}}) d t-\int_{a}^{b} f^{i}(t, \bar{y}, \dot{\bar{y}}) d t \\
& >\int_{a}^{b} \Phi\left(t, \bar{x}, \bar{x}, \bar{y}, \dot{\bar{y}} ;\left(f_{y}^{i}(t, \bar{y}, \dot{\bar{y}})-\frac{d}{d t}\left[f_{\dot{y}}^{i}(t, \bar{y}, \bar{y})\right], \rho_{f_{i}}\right)\right) d t, \quad i \in A .
\end{aligned}
$$

Multiplying both sides of the above inequalities by $\bar{\lambda}_{i}, i=1, \ldots, p$, where $\bar{\lambda} \geq 0$, $\bar{\lambda}^{T} e=1$, we obtain 


$$
\begin{aligned}
& \int_{a}^{b} \bar{\lambda}_{i} f^{i}(t, \bar{x}, \bar{x}) d t-\int_{a}^{b} \bar{\lambda}_{i} f^{i}(t, \bar{y}, \dot{\bar{y}}) d t \\
& \geqq \int_{a}^{b} \bar{\lambda}_{i} \Phi\left(t, \bar{x}, \bar{x}, \bar{y}, \bar{y} ;\left(f_{y}^{i}(t, \bar{y}, \bar{y})-\frac{d}{d t}\left[f_{\dot{y}}^{i}(t, \bar{y}, \bar{y})\right], \rho_{f_{i}}\right)\right) d t, \quad i \in A, \\
& \int_{a}^{b} \bar{\lambda}_{r} f^{r}(t, \bar{x}, \bar{x}) d t-\int_{a}^{b} \bar{\lambda}_{i} f^{r}(t, \bar{y}, \dot{y}) d t \\
& \quad>\int_{a}^{b} \bar{\lambda}_{r} \Phi\left(t, \bar{x}, \dot{\bar{x}}, \bar{y}, \dot{\bar{y}} ;\left(f_{y}^{r}(t, \bar{y}, \dot{\bar{y}})-\frac{d}{d t}\left[f_{\dot{y}}^{r}(t, \bar{y}, \bar{y})\right], \rho_{f_{i}}\right)\right) d t \\
& \text { for at least one } r \in A .
\end{aligned}
$$

Adding both sides of the inequalities (47) and (48), we get

$$
\begin{aligned}
& \int_{a}^{b} \sum_{i=1}^{p} \bar{\lambda}_{i} f^{i}(t, \bar{x}, \dot{\bar{x}}) d t-\int_{a}^{b} \sum_{i=1}^{p} \bar{\lambda}_{i} f^{i}(t, \bar{y}, \dot{\bar{y}}) d t \\
& >\int_{a}^{b} \sum_{i=1}^{p} \bar{\lambda}_{i} \Phi\left(t, \bar{x}, \dot{\bar{x}}, \bar{y}, \bar{y} ;\left(f_{y}^{i}(t, \bar{y}, \dot{\bar{y}})-\frac{d}{d t}\left[f_{\dot{y}}^{i}(t, \bar{y}, \bar{y})\right], \rho_{f_{i}}\right)\right) d t .
\end{aligned}
$$

By (46) and (47), it follows that the following inequality

$$
\int_{a}^{b} \sum_{i=1}^{p} \bar{\lambda}_{i} f^{i}(t, \bar{x}, \dot{\bar{x}}) d t>\int_{a}^{b} \sum_{i=1}^{p} \bar{\lambda}_{i} f^{i}(t, \bar{y}, \dot{\bar{y}}) d t
$$

holds, contradicting (27). Hence, $\bar{x}=\bar{y}$ and efficiency of $\bar{y}$ in the multiobjective variational control problem (MVPP) follows by the weak duality theorem (Theorem 8). Thus, the theorem is proved.

\section{Wolfe duality}

In this section, we prove duality results between the considered multiobjective variational programming problem (MVPP) and its Wolfe type vector variational control dual problem (VWD) defined as follows

$$
\begin{array}{r}
\text { Maximize } \int_{a}^{b}\left\{f(t, y(t), \dot{y}(t))+\xi(t)^{T} g(t, y(t), \dot{y}(t)) e\right. \\
\left.+\zeta(t)^{T} h(t, y(t), \dot{y}(t)) e\right\} d t
\end{array}
$$




$$
\begin{aligned}
& \text { subject to }\left[\lambda^{T} f_{y}(t, y(t), \dot{y}(t))+\xi(t)^{T} g_{y}(t, y(t), \dot{y}(t))\right. \\
& \left.+\zeta(t)^{T} h_{y}(t, y(t), \dot{y}(t))\right]=\frac{d}{d t}\left[\lambda^{T} f_{\dot{y}}(t, y(t), \dot{y}(t))+\xi(t)^{T} g_{\dot{y}}(t, y, \dot{y})\right. \\
& \left.+\zeta(t)^{T} h_{\dot{y}}(t, y, \dot{y})\right], t \in I, \\
& x(a)=\alpha, \quad x(b)=\beta, \\
& \lambda \geq 0, \quad \lambda^{T} e=1, \quad \xi(t) \geqq 0,
\end{aligned}
$$

where $e=(1, \ldots, 1) \in R^{p}$ is a $p$-dimensional vector. It may be noted here that the above dual constraints are written using the Karush-Kuhn-Tucker necessary optimality conditions for the problem (MVPP).

Let $\Omega_{W}$ be the set of all feasible solutions $(y, \lambda, \xi, \zeta)$ in Wolfe type dual problem (VWD). We denote by $Y$ the set $Y=\left\{y \in X:(y, \lambda, \xi, \zeta) \in \Omega_{W}\right\}$.

Theorem 14 (Weak duality): Let $x$ and $(y, \lambda, \xi, \zeta)$ be any feasible solutions in problems (MVPP) and (VWD). Further, assume that the following hypotheses are fulfilled:

(a) $\int_{a}^{b} f^{i}(t, \cdot, \cdot) d t, i=1, \ldots, k$, is strictly $\left(\Phi, \rho_{f_{i}}\right)$-invex at y on $S \cup Y$,

(b) $\int_{a}^{b} g^{j}(t, \cdot, \cdot) d t, j=1, \ldots, q$, is $\left(\Phi, \rho_{g_{j}}\right)$-invex at y on $S \cup Y$,

(c) $\int_{a}^{b} h^{k}(t, \cdot, \cdot) d t, k \in K^{+}(t)=\left\{k \in K: \zeta_{k}(t)>0\right\}$, is $\left(\Phi, \rho_{h_{k}}\right)$-invex at y on $S \cup Y$,

(d) $\int_{a}^{b}\left(-h^{k}(t, \cdot, \cdot)\right) d t, k \in K^{-}(t)=\left\{k \in K: \zeta_{k}(t)<0\right\}$, is $\left(\Phi, \rho_{h_{k}}\right)$-invex at y on $S \cup Y$,

(e) $\sum_{i=1}^{p} \lambda_{i} \rho_{f_{i}}+\sum_{j=1}^{q} \xi_{j} \rho_{g_{j}}+\sum_{k \in K^{+}(t)} \zeta_{k} \rho_{h_{k}}-\sum_{k \in K^{-}(t)} \zeta_{k} \rho_{h_{k}} \geqq 0$.

Then, the following cannot hold

$$
\int_{a}^{b} f(t, x, \dot{x}) d t \leq \int_{a}^{b}\left\{f(t, y, \dot{y})+\xi(t)^{T} g(t, y, \dot{y}) e+\zeta(t)^{T} h(t, y, \dot{y}) e\right\} d t
$$

that is, the following cannot hold

$$
\begin{gathered}
\int_{a}^{b} f(t, x, \dot{x}) d t \leqq \int_{a}^{b}\left\{f(t, y, \dot{y})+\xi(t)^{T} g(t, y, \dot{y}) e+\zeta(t)^{T} h(t, y, \dot{y}) e\right\} d t \\
\forall i \in A
\end{gathered}
$$

and

$$
\int_{a}^{b} f^{r}(t, x, \dot{x}) d t<\int_{a}^{b}\left(f^{r}(t, y, \dot{y})+\xi(t)^{T} g(t, y, \dot{y})+\zeta(t)^{T} h(t, y, \dot{y})\right) d t
$$

for at least one $r \in A$. 
Proof Suppose, contrary to the result of the theorem, that the inequalities (50) and (51) are satisfied. Since $\lambda \geq 0, \lambda^{T} e=1$, the inequalities (50) and (51) yield

$$
\begin{gathered}
\int_{a}^{b} \sum_{i=1}^{p} \lambda_{i} f^{i}(t, x, \dot{x}) d t \leqq \int_{a}^{b} \sum_{i=1}^{p} \lambda_{i} f^{i}(t, y, \dot{y}) d t+\int_{a}^{b} \sum_{j=1}^{q} \xi_{j} g^{j}(t, y, \dot{y}) d t \\
+\int_{a}^{b} \sum_{k=1}^{s} \zeta_{k} h^{k}(t, y, \dot{y}) d t \leqq 0 .
\end{gathered}
$$

By assumption, the hypotheses (a)-(d) are fulfilled. Thus, by Definition 3, we have

$$
\begin{aligned}
& \int_{a}^{b} f^{i}(t, x, \dot{x}) d t-\int_{a}^{b} f^{i}(t, y, \dot{y}) d t \\
& \quad>\int_{a}^{b} \Phi\left(t, x, \dot{x}, y, \dot{y} ;\left(f_{y}^{i}(t, y, \dot{y})-\frac{d}{d t}\left[f_{\dot{y}}^{i}(t, y, \dot{y})\right], \rho_{f_{i}}\right]\right) d t, \quad i \in A,
\end{aligned}
$$$$
\int_{a}^{b} g^{j}(t, x, \dot{x}) d t-\int_{a}^{b} g^{j}(t, y, \dot{y}) d t
$$$$
\geqq \int_{a}^{b} \Phi\left(t, x, \dot{x}, y, \dot{y} ;\left(g_{y}^{j}(t, y, \dot{y})-\frac{d}{d t}\left[g_{\dot{y}}^{j}(t, y, \dot{y})\right], \rho_{g_{j}}\right)\right) d t, \quad j \in J,
$$

$\int_{a}^{b} h^{k}(t, x, \dot{x}) d t-\int_{a}^{b} h^{k}(t, y, \dot{y}) d t$

$$
\geqq \int_{a}^{b} \Phi\left(t, x, \dot{x}, y, \dot{y} ;\left(h_{y}^{k}(t, y, \dot{y})-\frac{d}{d t}\left[h_{y}^{k}(t, y, \dot{y})\right], \rho_{h_{k}}\right)\right) d t, \quad k \in K^{+}(t),
$$$$
-\int_{a}^{b} h^{k}(t, x, \dot{x}) d t+\int_{a}^{b} h^{k}(t, y, \dot{y}) d t
$$$$
\geqq-\int_{a}^{b} \Phi\left(t, x, \dot{x}, y, \dot{y} ;\left(-h_{y}^{k}(t, y, \dot{y})-\frac{d}{d t}\left[-h_{y}^{k}(t, y, \dot{y})\right], \rho_{h_{k}}\right)\right) d t, k \in K^{-}(t) .
$$

Using the last constraint of (VWD) together with (53)-(56), we get

$$
\begin{aligned}
& \int_{a}^{b} \lambda_{i} f^{i}(t, x, \dot{x}) d t-\int_{a}^{b} \lambda_{i} f^{i}(t, y, \dot{y}) d t \\
& \quad>\int_{a}^{b} \lambda_{i} \Phi\left(t, x, \dot{x}, y, \dot{y} ;\left(f_{y}^{i}(t, y, \dot{y})-\frac{d}{d t}\left[f_{\dot{y}}^{i}(t, y, \dot{y})\right], \rho_{f_{i}}\right)\right) d t, \quad i \in A,
\end{aligned}
$$




$$
\begin{aligned}
& \int_{a}^{b} \lambda_{r} f^{r}(t, x, \dot{x}) d t-\int_{a}^{b} \lambda_{r} f^{r}(t, y, \dot{y}) d t \\
& \quad>\int_{a}^{b} \lambda_{r} \Phi\left(t, x, \dot{x}, y, \dot{y} ;\left(f_{y}^{r}(t, y, \dot{y})-\frac{d}{d t}\left[f_{\dot{y}}^{r}(t, y, \dot{y})\right], \rho_{f_{r}}\right)\right) d t
\end{aligned}
$$

for at least one $r \in A$,

$$
\begin{aligned}
& \int_{a}^{b} \xi_{j} g^{j}(t, x, \dot{x}) d t-\int_{a}^{b} \xi_{j} g^{j}(t, y, \dot{y}) d t \\
& \quad \geqq \int_{a}^{b} \xi_{j} \Phi\left(t, x, \dot{x}, y, \dot{y} ;\left(g_{y}^{j}(t, y, \dot{y})-\frac{d}{d t}\left[g_{\dot{y}}^{j}(t, y, \dot{y})\right], \rho_{g_{j}}\right)\right) d t, \quad j \in J,
\end{aligned}
$$

$$
\int_{a}^{b} \zeta_{k} h^{k}(t, x, \dot{x}) d t-\int_{a}^{b} \zeta_{k} h^{k}(t, y, \dot{y}) d t
$$

$$
\geqq \int_{a}^{b} \zeta_{k} \Phi\left(t, x, \dot{x}, y, \dot{y} ;\left(h_{y}^{k}(t, y, \dot{y})-\frac{d}{d t}\left[h_{\dot{y}}^{k}(t, y, \dot{y})\right], \rho_{h_{k}}\right)\right) d t, \quad k \in K^{+}(t),
$$

$$
\int_{a}^{b} \zeta_{k} h^{k}(t, x, \dot{x}) d t-\int_{a}^{b} \zeta_{k} h^{k}(t, y, \dot{y}) d t
$$

$$
\begin{array}{r}
\geqq \int_{a}^{b}\left(-\zeta_{k}\right) \Phi\left(t, x, \dot{x}, y, \dot{y} ;\left(-h_{y}^{k}(t, y, \dot{y})-\frac{d}{d t}\left[-h_{y}^{k}(t, y, \dot{y})\right], \rho_{h_{k}}\right)\right) d t, \\
k \in K^{-}(t) .
\end{array}
$$

Adding both sides of the inequalities (59)-(61) and then, using the feasibility of $x$ in problem (MVPP) and the feasibility of $(y, \lambda, \xi, \zeta)$ in problem (VWD), we obtain, respectively,

$$
\begin{aligned}
0 \geqq & \int_{a}^{b} \sum_{j=1}^{q} \xi_{j} g^{j}(t, y, \dot{y}) d t \\
& +\int_{a}^{b} \sum_{j=1}^{q} \xi_{j} \Phi\left(t, x, \dot{x}, y, \dot{y} ; g_{y}^{j}(t, y, \dot{y})-\frac{d}{d t}\left[g_{y}^{j}(t, y, \dot{y})\right], \rho_{g_{j}}\right) d t, \quad j \in J,
\end{aligned}
$$




$$
\begin{aligned}
0 & \geqq \int_{a}^{b} \sum_{k \in K} \zeta_{k} h^{k}(t, y, \dot{y}) d t \\
& +\int_{a}^{b} \sum_{k \in K^{+}(t)} \zeta_{k} \Phi\left(t, x, \dot{x}, y, \dot{y} ;\left(h_{y}^{k}(t, y, \dot{y})-\frac{d}{d t}\left[h_{\dot{y}}^{k}(t, y, \dot{y})\right], \rho_{h_{k}}\right)\right) d t \\
& +\int_{a}^{b} \sum_{k \in K^{-}(t)}\left(-\zeta_{k}\right) \Phi\left(t, x, \dot{x}, y, \dot{y} ;\left(-h_{y}^{k}(t, y, \dot{y})-\frac{d}{d t}\left[-h_{y}^{k}(t, y, \dot{y})\right], \rho_{h_{k}}\right)\right) d t .
\end{aligned}
$$

Combining (57), (58), (62) and, (63), we get

$$
\begin{aligned}
& \int_{a}^{b} \sum_{i=1}^{p} \lambda_{i} f^{i}(t, x, \dot{x}) d t>\int_{a} \sum_{i=1}^{p} \lambda_{i} f^{i}(t, y, \dot{y}) d t \\
& +\int_{a}^{b} \sum_{j=1}^{q} \xi_{j} g^{j}(t, y, \dot{y}) d t+\int_{a} \sum_{k=1}^{s} \zeta_{k} h^{k}(t, y, \dot{y}) d t \\
& +\int_{a}^{b} \sum_{i=1}^{p} \lambda_{i} \Phi\left(t, x, \dot{x}, y, \dot{y} ;\left(f_{y}^{i}(t, y, \dot{y})-\frac{d}{d t}\left[f_{\dot{y}}^{i}(t, y, \dot{y})\right], \rho_{f_{i}}\right)\right) d t \\
& +\int_{a}^{b} \sum_{j=1}^{q} \xi_{j} \Phi\left(t, x, \dot{x}, y, \dot{y} ;\left(g_{y}^{j}(t, y, \dot{y})-\frac{d}{d t}\left[g_{\dot{y}}^{j}(t, y, \dot{y})\right], \rho_{g_{j}}\right)\right) d t \\
& +\int_{a}^{b} \sum_{k \in K^{+}(t)} \zeta_{k} \Phi\left(t, x, \dot{x}, y, \dot{y} ;\left(h_{y}^{k}(t, y, \dot{y})-\frac{d}{d t}\left[h_{\dot{y}}^{k}(t, y, \dot{y})\right], \rho_{h_{k}}\right)\right) d t \\
& +\int_{a}^{b} \sum_{k \in K^{-}(t)}\left(-\zeta_{k}\right) \Phi\left(t, x, \dot{x}, y, \dot{y} ;\left(-h_{y}^{k}(t, y, \dot{y})-\frac{d}{d t}\left[-h_{\dot{y}}^{k}(t, y, \dot{y})\right], \rho_{h_{k}}\right)\right) d t .
\end{aligned}
$$

By (52) and (64), it follows that

$$
\begin{aligned}
& \int_{a}^{b} \sum_{i=1}^{p} \lambda_{i} \Phi\left(t, x, \dot{x}, y, \dot{y} ;\left(f_{y}^{i}(t, y, \dot{y})-\frac{d}{d t}\left[f_{\dot{y}}^{i}(t, y, \dot{y})\right], \rho_{f_{i}}\right)\right) d t \\
& \quad+\int_{a}^{b} \sum_{j=1}^{q} \xi_{j} \Phi\left(t, x, \dot{x}, y, \dot{y} ;\left(g_{y}^{j}(t, y, \dot{y})-\frac{d}{d t}\left[g_{\dot{y}}^{j}(t, y, \dot{y})\right], \rho_{g_{j}}\right)\right) d t
\end{aligned}
$$




$$
\begin{aligned}
& +\int_{a}^{b} \sum_{k \in K^{+}(t)} \zeta_{k} \Phi\left(t, x, \dot{x}, y, \dot{y} ;\left(h_{y}^{k}(t, y, \dot{y})-\frac{d}{d t}\left[h_{y}^{k}(t, y, \dot{y})\right], \rho_{h_{k}}\right)\right) d t \\
& \int_{a}^{b} \sum_{k \in K^{-}(t)}\left(-\zeta_{k}\right) \Phi\left(t, x, \dot{x}, y, \dot{y} ;\left(-h_{y}^{k}(t, y, \dot{y})-\frac{d}{d t}\left[-h_{\dot{y}}^{k}(t, y, \dot{y})\right], \rho_{h_{k}}\right)\right) d t<0 .
\end{aligned}
$$

We denote

$$
\begin{aligned}
\tilde{\lambda}_{i} & =\frac{\lambda_{i}}{\sum_{i=1}^{p} \lambda_{i}+\sum_{j=1}^{q} \xi_{j}(t)+\sum_{k \in K^{+}(t)} \zeta_{k}(t)-\sum_{k \in K^{-}(t)} \zeta_{k}(t)}, \quad i \in A, \\
\widetilde{\xi}_{j}(t) & =\frac{\xi_{j}(t)}{\sum_{i=1}^{p} \lambda_{i}+\sum_{j=1}^{q} \xi_{j}(t)+\sum_{k \in K^{+}(t)} \zeta_{k}(t)-\sum_{k \in K^{-}(t)} \zeta_{k}(t)}, \quad j \in J, \\
\tilde{\zeta}_{k}(t) & =\frac{\zeta_{k}(t)}{\sum_{i=1}^{p} \lambda_{i}+\sum_{j=1}^{q} \xi_{j}(t)+\sum_{k \in K^{+}(t)} \zeta_{k}(t)-\sum_{k \in K^{-}(t)} \zeta_{k}(t)}, \quad k \in K^{+}(t), \\
\tilde{\zeta}_{k}(t) & =\frac{-\zeta_{k}(t)}{\sum_{i=1}^{p} \lambda_{i}+\sum_{j=1}^{q} \xi_{j}(t)+\sum_{k \in K^{+}(t)} \zeta_{k}(t)-\sum_{k \in K^{-}(t)} \zeta_{k}(t)}, \quad k \in K^{-}(t) .
\end{aligned}
$$

By (66)-(69), it follows that

$$
\sum_{i=1}^{p} \widetilde{\lambda}_{i}+\sum_{j=1}^{q} \widetilde{\xi}_{j}(t)+\sum_{k \in K^{+}(t)} \widetilde{\zeta}_{k}(t)+\sum_{k \in K^{-}(t)} \widetilde{\zeta}_{k}(t)=1 .
$$

Combining (65)-(69), we get

$$
\begin{aligned}
& \int_{a}^{b} \sum_{i=1}^{p} \widetilde{\lambda}_{i} \Phi\left(t, x, \dot{x}, y, \dot{y} ;\left(f_{y}^{i}(t, y, \dot{y})-\frac{d}{d t}\left[f_{\dot{y}}^{i}(t, y, \dot{y})\right], \rho_{f_{i}}\right)\right) d t \\
& \quad+\int_{a}^{b} \sum_{j=1}^{q} \widetilde{\xi}_{j} \Phi\left(t, x, \dot{x}, y, \dot{y} ;\left(g_{y}^{j}(t, y, \dot{y})-\frac{d}{d t}\left[g_{\dot{y}}^{j}(t, y, \dot{y})\right], \rho_{g_{j}}\right)\right) d t \\
& \quad+\int_{a}^{b} \sum_{k \in K^{+}(t)} \widetilde{\zeta}_{k} \Phi\left(t, x, \dot{x}, y, \dot{y} ;\left(h_{y}^{k}(t, y, \dot{y})-\frac{d}{d t}\left[h_{\dot{y}}^{k}(t, y, \dot{y})\right], \rho_{h_{k}}\right)\right) d t \\
& \quad+\int_{a}^{b} \sum_{k \in K^{-}(t)} \widetilde{\zeta}_{k} \Phi\left(t, x, \dot{x}, y, \dot{y} ;\left(-h_{y}^{k}(t, y, \dot{y})-\frac{d}{d t}\left[-h_{\dot{y}}^{k}(t, y, \dot{y})\right], \rho_{h_{k}}\right)\right) d t<0 .
\end{aligned}
$$

By Definition 3, it follows that the functional $\Phi(t, x, \dot{x}, y, \dot{y} ; \cdot)$ is convex on $R^{n+1}$. Thus, by (71) and (70), Definition 2 implies 


$$
\begin{aligned}
& \int_{a}^{b} \Phi\left(t, x, \dot{x}, y, \dot{y} ;\left(\sum_{i=1}^{p} \widetilde{\lambda}_{i} f_{y}^{i}(t, y, \dot{y})+\sum_{j=1}^{q} \widetilde{\xi}_{j} g_{y}^{j}(t, y, \dot{y})\right.\right. \\
& +\sum_{k \in K^{+}(t)} \widetilde{\zeta}_{k} h_{y}^{k}(t, y, \dot{y})+\sum_{k \in K^{-}(t)}\left(-\widetilde{\zeta}_{k}\right) h_{y}^{k}(t, y, \dot{y}) \\
& \quad-\frac{d}{d t}\left[\sum_{i=1}^{p} \widetilde{\lambda}_{\dot{y}}^{i}(t, y, \dot{y})+\sum_{j=1}^{q} \widetilde{\xi}_{j} g_{\dot{y}}^{j}(t, y, \dot{y})+\sum_{k \in K^{+}(t)} \widetilde{\zeta}_{k} h_{\dot{y}}^{k}(t, y, \dot{y})\right. \\
& \left.\left.\left.\quad+\sum_{k \in K^{-}(t)}\left(-\widetilde{\zeta}_{k}\right) h_{\dot{y}}^{k}(t, y, \dot{y})\right], \sum_{i=1}^{p} \widetilde{\lambda} \rho_{f_{i}}+\sum_{j=1}^{q} \widetilde{\xi}_{j} \rho_{g_{j}}+\sum_{k \in K^{+}(t) \cup K^{-}(t)} \widetilde{\zeta}_{k} \rho_{h_{k}}\right)\right) d t<0 .
\end{aligned}
$$

Hence, the first constraint of (VWD) yields

$$
\int_{a}^{b} \Phi\left(t, x, \dot{x}, y, \dot{y} ;\left(0, \sum_{i=1}^{p} \widetilde{\lambda}_{i} \rho_{f_{i}}+\sum_{j=1}^{q} \widetilde{\xi}_{j}(t) \rho_{g_{j}}+\sum_{k \in K^{+}(t) \cup K^{-}(t)} \widetilde{\zeta}_{k}(t) \rho_{h_{k}}\right)\right) d t<0 .
$$

From the hypothesis (e), it follows that

$$
\sum_{i=1}^{p} \widetilde{\lambda}_{i} \rho_{f_{i}}+\sum_{j=1}^{q} \widetilde{\xi}_{j}(t) \rho_{g_{j}}+\sum_{k=1}^{s} \widetilde{\zeta}_{k}(t) \rho_{h_{k}} \geqq 0
$$

By Definition 3, it follows that $\Phi(t, x, \dot{x}, y, \dot{y},(0, a)) \geqq 0$ for any $a \in R_{+}$. Thus, (73) implies that the following inequality

$$
\int_{a}^{b} \Phi\left(t, x, \dot{x}, y, \dot{y} ;\left(0, \sum_{i=1}^{p} \tilde{\lambda}_{i} \rho f_{i}+\sum_{j=1}^{q} \widetilde{\xi}_{j}(t) \rho_{g_{j}}+\sum_{k \in K^{+}(t) \cup K^{-}(t)} \widetilde{\zeta}_{k}(t) \rho h_{k}\right)\right) d t \geqq 0
$$

holds, contradicting (72).

Theorem 15 (Strong duality): Let $\bar{x}$ be an efficient solution in the considered multiobjective variational control problem (MVPP). Further, assume that the Kuhn-Tucker constraint qualification is satisfied for $(M V P P)$. Then there exist $\bar{\lambda} \in R_{+}^{p}$ and the piecewise smooth functions $\bar{\xi}(\cdot): I \rightarrow R^{m}$ and $\bar{\zeta}(\cdot): I \rightarrow R^{s}$ such that $(\bar{x}, \bar{\lambda}, \bar{\xi}(t), \bar{\zeta}(t))$ is a feasible solution for problem (VWD). If also the weak duality theorem (Theorem 14) holds between (MVPP) and (VWD), then $(\bar{x}, \bar{\lambda}, \bar{\xi}(t), \bar{\zeta}(t))$ is an efficient solution in Wolfe type dual problem (VWD) and the objective function values are equal.

Proof By assumption, $\bar{x}$ is an efficient solution in the considered multiobjective variational control problem (MVPP). Hence, by Theorem 9, there exist $\bar{\lambda} \in R^{p}$ and the piecewise smooth functions $\bar{\xi}(\cdot): I \rightarrow R^{m}$ and $\bar{\zeta}(\cdot): I \rightarrow R^{s}$ such that the KarushKuhn-Tucker optimality conditions (24)-(26) are satisfied. Thus, $(\bar{x}, \bar{\lambda}, \bar{\xi}(t), \bar{\zeta}(t))$ is 
a feasible solution in Wolfe dual problem (VWD) and the two objective functionals have the same values.

Now, we show that $(\bar{x}, \bar{\lambda}, \bar{\xi}(t), \bar{\zeta}(t))$ is an efficient solution in Wolfe type dual problem (VWD). We proceed by contradiction. Suppose that $(\bar{x}, \bar{\lambda}, \bar{\xi}(t), \bar{\zeta}(t))$ is not efficient in problem (VWD). Then, there exists $(\widehat{x}, \widehat{\lambda}, \widehat{\xi}(t), \widehat{\zeta}(t)) \in \Omega_{W}$ such that

$$
\begin{aligned}
& \int_{a}^{b}\left\{f^{i}(t, \widehat{x}, \dot{\hat{x}})+\widehat{\xi}(t)^{T} g(t, \widehat{x}, \dot{\hat{x}})+\widehat{\zeta}(t)^{T} h(t, \widehat{x}, \dot{\hat{x}})\right\} d t \\
& \quad \geqq \int_{a}^{b}\left\{f^{i}(t, \bar{x}, \dot{\bar{x}})+\bar{\xi}(t)^{T} g(t, \bar{x}, \dot{\bar{x}})+\bar{\zeta}(t)^{T} h(t, \bar{x}, \dot{\bar{x}})\right\} d t, \quad i \in A,
\end{aligned}
$$

and

$$
\begin{aligned}
& \int_{a}^{b}\left\{f^{r}(t, \widehat{x}, \dot{\widehat{x}})+\widehat{\xi}(t)^{T} g(t, \widehat{x}, \dot{\hat{x}})+\widehat{\zeta}(t)^{T} h(t, \widehat{x}, \dot{\widehat{x}})\right\} d t>0 \\
& \int_{a}^{b}\left\{f^{r}(t, \bar{x}, \dot{\bar{x}})+\bar{\xi}(t)^{T} g(t, \bar{x}, \dot{\bar{x}})+\bar{\zeta}(t)^{T} h(t, \bar{x}, \dot{\bar{x}})\right\} d t \quad \text { for some } r \in A .
\end{aligned}
$$

Using the feasibility of $\bar{x}$ in problem (MVPP) together with (25), we get

$$
\int_{a}^{b}\left\{f^{i}(t, \widehat{x}, \dot{\widehat{x}})+\widehat{\xi}(t)^{T} g(t, \widehat{x}, \dot{\widehat{x}})+\widehat{\zeta}(t)^{T} h(t, \widehat{x}, \dot{\widehat{x}})\right\} d t \geqq \int_{a}^{b} f^{i}(t, \bar{x}, \dot{\bar{x}}) d t, \quad i \in A,
$$

and

$$
\begin{aligned}
& \int_{a}^{b}\left\{f^{r}(t, \widehat{x}, \dot{\hat{x}})+\widehat{\xi}(t)^{T} g(t, \widehat{x}, \dot{\widehat{x}})+\widehat{\zeta}(t)^{T} h(t, \widehat{x}, \dot{\widehat{x}})\right\} d t \\
& >\int_{a}^{b} f^{r}(t, \bar{x}, \dot{\bar{x}}) d t, \text { for some } r \in A .
\end{aligned}
$$

The inequalities above contradict the weak duality theorem (Theorem 14). Thus, $(\bar{x}, \bar{\lambda}, \bar{\xi}(t), \bar{\zeta}(t))$ is efficient in problem (VWD).

Proposition 16 Let $(\bar{x}, \bar{\lambda}, \bar{\xi}(t), \bar{\zeta}(t))$ be a feasible solution in Wolfe type dual problem $(V W D)$ such that $\bar{x} \in S$. Further, assume that the following hypotheses are fulfilled:

(a) $\int_{a}^{b} f^{i}(t, \cdot, \cdot) d t, i=1, \ldots, k$, is strictly $\left(\Phi, \rho_{f_{i}}\right)$-invex at $\bar{x}$ on $S$,

(b) $\int_{a}^{b} g^{j}(t, \cdot, \cdot) d t, j=1, \ldots, q$, is $\left(\Phi, \rho_{g_{j}}\right)$-invex at $\bar{x}$ on $S$,

(c) $\int_{a}^{b} h^{k}(t, \cdot, \cdot) d t, k \in K^{+}(t)=\left\{k \in K: \bar{\zeta}_{k}(t)>0\right\}$, is ( $\left.\Phi, \rho_{h_{k}}\right)$-invex at $\bar{x}$ on $S$, 
(d) $\int_{a}^{b}\left(-h^{k}(t, \cdot, \cdot)\right) d t, k \in K^{-}(t)=\left\{k \in K: \bar{\zeta}_{k}(t)<0\right\}$, is $\left(\Phi, \rho_{h_{k}}\right)$-invex at $\bar{x}$ on $S$

(e) $\sum_{i=1}^{p} \bar{\lambda}_{i} \rho_{f_{i}}+\sum_{j=1}^{q} \bar{\xi}_{j} \rho_{g_{j}}+\sum_{k \in K^{+}(t)} \bar{\zeta}_{k} \rho_{h_{k}}-\sum_{k \in K^{-}(t)} \bar{\zeta}_{k} \rho_{h_{k}} \geqq 0$.

Then $\bar{x}$ is efficient in the considered multiobjective variational control problem $(M V P P)$.

Theorem 17 (Converse duality): Let $(\bar{x}, \bar{\lambda}, \bar{\xi}(t), \bar{\zeta}(t))$ be an efficient solution in Wolfe type dual problem (VWD) and $\bar{x} \in S$. Further, assume that the hypotheses (a)-(e) of Proposition 16 are fulfilled. Then $\bar{x}$ is efficient in the considered multiobjective variational control problem (MVPP).

Proof Proof follows directly from Proposition 16.

Theorem 18 (Strict converse duality): Let $\bar{x}$ and $(\bar{y}, \bar{\lambda}, \bar{\xi}(t), \bar{\zeta}(t))$ be any feasible solutions in problems (MVPP) and (VMWD), respectively, such that

$$
\begin{aligned}
\int_{a}^{b} \sum_{i=1}^{p} \bar{\lambda}_{i} f^{i}(t, \bar{x}, \dot{\bar{x}}) d t \leqq & \int_{a}^{b} \sum_{i=1}^{p} \bar{\lambda}_{i} f^{i}(t, \bar{y}, \dot{\bar{y}}) d t+\int_{a}^{b} \sum_{j=1}^{q} \bar{\xi}_{j} g^{j}(t, \bar{y}, \dot{\bar{y}}) d t \\
& +\int_{a}^{b} \sum_{k \in K} \bar{\zeta}_{k} h^{k}(t, \bar{y}, \dot{\bar{y}}) d t .
\end{aligned}
$$

Further, assume that the following hypotheses are fulfilled:

(a) $\int_{a}^{b} f^{i}(t, \cdot, \cdot) d t, i=1, \ldots, k$, is strictly $\left(\Phi, \rho_{f_{i}}\right)$-invex at $\bar{y}$ on $S \cup Y$,

(b) $\int_{a}^{b} g^{j}(t, \cdot, \cdot) d t, j=1, \ldots, q$, is $\left(\Phi, \rho_{g_{j}}\right)$-invex at $\bar{y}$ on $S \cup Y$,

(c) $\int_{a}^{b} h^{k}(t, \cdot, \cdot) d t, k \in K^{+}(t)=\left\{k \in K: \bar{\zeta}_{k}(t)>0\right\}$, is $\left(\Phi, \rho_{h_{k}}\right)$-invex at $\bar{y}$ on $S \cup Y$,

(d) $\int_{a}^{b}\left(-h^{k}(t, \cdot, \cdot)\right) d t, k \in K^{-}(t)=\left\{k \in K: \bar{\zeta}_{k}(t)<0\right\}$, is $\left(\Phi, \rho_{h_{k}}\right)$-invex at $\bar{y}$ on $S \cup Y$

(e) $\sum_{i=1}^{p} \bar{\lambda}_{i} \rho_{f_{i}}+\sum_{j=1}^{q} \bar{\xi}_{j} \rho_{g_{j}}+\sum_{k \in K^{+}(t)} \bar{\zeta}_{k} \rho_{h_{k}}-\sum_{k \in K^{-}(t)} \bar{\zeta}_{k} \rho_{h_{k}} \geqq 0$.

Then $\bar{x}=\bar{y}$ and $\bar{y}$ is efficient in the considered multiobjective variational control problem (MVPP).

Proof Suppose, contrary to the result, that $\bar{x} \neq \bar{y}$. By hypotheses (a)-(d), Definition 3 gives

$$
\begin{aligned}
& \int_{a}^{b} f^{i}(t, \bar{x}, \dot{\bar{x}}) d t-\int_{a}^{b} f^{i}(t, \bar{y}, \dot{\bar{y}}) d t \\
& \quad>\int_{a}^{b} \Phi\left(t, \bar{x}, \dot{\bar{x}}, \bar{y}, \dot{\bar{y}} ;\left(f_{y}^{i}(t, \bar{y}, \dot{\bar{y}})-\frac{d}{d t}\left[f_{\dot{y}}^{i}(t, \bar{y}, \dot{\bar{y}})\right], \rho_{f_{i}}\right)\right) d t
\end{aligned}
$$




$$
\begin{aligned}
& \int_{a}^{b} g^{j}(t, \bar{x}, \dot{\bar{x}}) d t-\int_{a}^{b} g^{j}(t, \bar{y}, \dot{\bar{y}}) d t \\
& \quad \geqq \int_{a}^{b} \Phi\left(t, \bar{x}, \dot{\bar{x}}, \bar{y}, \dot{\bar{y}} ;\left(g_{y}^{j}(t, \bar{y}, \dot{\bar{y}})-\frac{d}{d t}\left[g_{\dot{y}}^{j}(t, \bar{y}, \dot{\bar{y}})\right], \rho_{g_{j}}\right)\right) d t, \quad j \in J, \\
& \int_{a}^{b} h^{k}(t, \bar{x}, \dot{\bar{x}}) d t-\int_{a}^{b} h^{k}(t, \bar{y}, \dot{\bar{y}}) d t \\
& \geqq \int_{a}^{b} \Phi\left(t, \bar{x}, \dot{\bar{x}}, \bar{y}, \dot{\bar{y}}\left(h_{y}^{k}(t, \bar{y}, \dot{\bar{y}})-\frac{d}{d t}\left[h_{\dot{y}}^{k}(t, \bar{y}, \dot{\bar{y}})\right], \rho_{h_{k}}\right)\right) d t, \quad k \in K^{+}(t) \\
& -\int_{a}^{b} h^{k}(t, \bar{x}, \dot{\bar{x}}) d t+\int_{a}^{b} h^{k}(t, \bar{y}, \dot{\bar{y}}) d t \\
& \geqq \int_{a} \Phi\left(t, \bar{x}, \dot{\bar{x}}, \bar{y}, \dot{\bar{y}} ;\left(-h_{y}^{k}(t, \bar{y}, \dot{\bar{y}})-\frac{d}{d t}\left[-h_{\dot{y}}^{k}(t, \bar{y}, \dot{\bar{y}})\right], \rho_{h_{k}}\right)\right) d t, \quad k \in K^{-}(t) .
\end{aligned}
$$

Hence, by $\bar{x} \in S$ and $(\bar{y}, \bar{\lambda}, \bar{\xi}(t), \bar{\zeta}(t)) \in \Omega_{W},(75)-(78)$ yield

$$
\begin{aligned}
& \int_{a}^{b} \sum_{i=1}^{p} \bar{\lambda}_{i} f^{i}(t, \bar{x}, \dot{\bar{x}}) d t-\left[\int_{a}^{b} \sum_{i=1}^{p} \bar{\lambda}_{i} f^{i}(t, \bar{y}, \dot{y}) d t\right. \\
& \left.+\int_{a}^{b} \sum_{j=1}^{q} \bar{\xi}_{j} g^{j}(t, \bar{y}, \dot{y}) d t \int_{a}^{b} \sum_{k \in K^{+}(t)} \bar{\zeta}_{k} h^{k}(t, \bar{y}, \dot{y}) d t+\int_{a}^{b} \sum_{k \in K^{-}(t)} \bar{\zeta}_{k} h^{k}(t, \bar{y}, \dot{y}) d t\right] \\
& >\int_{a}^{b} \sum_{i=1}^{p} \bar{\lambda}_{i} \Phi\left(t, \bar{x}, \dot{x}, \bar{y}, \dot{y} ;\left(f_{y}^{i}(t, \bar{y}, \dot{y})-\frac{d}{d t}\left[f_{\dot{y}}^{i}(t, \bar{y}, \bar{y})\right], \rho_{f_{i}}\right)\right) d t \\
& +\int_{a}^{b} \sum_{j=1}^{q} \bar{\xi}_{j} \Phi\left(t, \bar{x}, \dot{x}, \bar{y}, \dot{y} ;\left(g_{y}^{j}(t, \bar{y}, \dot{y})-\frac{d}{d t}\left[g_{\dot{y}}^{j}(t, \bar{y}, \dot{y})\right], \rho_{g_{j}}\right)\right) d t \\
& +\int_{a}^{b} \sum_{k \in K^{+}(t)} \bar{\zeta}_{k} \Phi\left(t, \bar{x}, \bar{x}, \bar{y}, \dot{y} ;\left(h_{y}^{k}(t, \bar{y}, \dot{y})-\frac{d}{d t}\left[h_{y}^{k}(t, \bar{y}, \bar{y})\right], \rho_{h_{k}}\right)\right) d t \\
& +\int_{a}^{b} \sum_{k \in K^{-}(t)} \bar{\zeta}_{k} \Phi\left(t, \bar{x}, \dot{\bar{x}}, \bar{y}, \bar{y} ;\left(-h_{y}^{k}(t, \bar{y}, \dot{\bar{y}})-\frac{d}{d t}\left[-h_{y}^{k}(t, \bar{y}, \dot{\bar{y}})\right], \rho_{h_{k}}\right)\right) d t .
\end{aligned}
$$


Combining (74) and (79), we get

$$
\begin{aligned}
& \int_{a}^{b} \sum_{i=1}^{p} \bar{\lambda}_{i} \Phi\left(t, \bar{x}, \dot{x}, \bar{y}, \dot{y} ;\left(f_{y}^{i}(t, \bar{y}, \dot{y})-\frac{d}{d t}\left[f_{\dot{y}}^{i}(t, \bar{y}, \dot{y})\right], \rho_{f_{i}}\right)\right) d t \\
& \quad+\int_{a}^{b} \sum_{j=1}^{q} \bar{\xi}_{j} \Phi\left(t, \bar{x}, \bar{x}, \bar{y}, \dot{y} ;\left(g_{y}^{j}(t, \bar{y}, \dot{y})-\frac{d}{d t}\left[g_{\dot{y}}^{j}(t, \bar{y}, \dot{y})\right], \rho_{g_{j}}\right)\right) d t \\
& \quad+\int_{a}^{b} \sum_{k \in K^{+}(t)} \bar{\zeta}_{k} \Phi\left(t, \bar{x}, \dot{x}, \bar{y}, \dot{y} ;\left(h_{y}^{k}(t, \bar{y}, \bar{y})-\frac{d}{d t}\left[h_{\dot{y}}^{k}(t, \bar{y}, \dot{y})\right], \rho_{h_{k}}\right)\right) d t \\
& \quad+\int_{a}^{b} \sum_{k \in K^{-}(t)} \bar{\zeta}_{k} \Phi\left(t, \bar{x}, \bar{x}, \bar{y}, \bar{y} ;\left(-h_{y}^{k}(t, \bar{y}, \dot{y})-\frac{d}{d t}\left[-h_{\dot{y}}^{k}(t, \bar{y}, \dot{y})\right], \rho_{h_{k}}\right)\right) d t<0 .
\end{aligned}
$$

The last part of proof is similar to the proof of Theorem 14.

\section{Conclusion}

Many works of variational problems have been focused on looking for solutions for these problems from duality results, studying the properties of the classes of functionals which are involved, and from the relationship between multiobjective variational programming problems and their duals.

In this paper, the concept of $(\Phi, \rho)$-invexity was extended to multiobjective variational control problems. Further, the concept of efficiency has been used to formulate multiobjective variational control dual problems in the sense of Mond-Weir and in the sense of Wolfe for the considered multiobjective variational programming problem. Under $(\Phi, \rho)$-invexity assumptions imposed on the functions involved in the considered multiobjective variational control problem, weak, strong, converse and strict converse dual theorems in the sense of Mond-Weir and in the sense of Wolfe have been proved between a new class of multiobjective variational control problems and their duals. Since $(\Phi, \rho)$-invexity notion unifies several concepts of generalized convex functions, therefore, the results established in the paper extend duality results for multiobjective variational control problems in a fairly large number of earlier works.

Open Access This article is distributed under the terms of the Creative Commons Attribution License which permits any use, distribution, and reproduction in any medium, provided the original author(s) and the source are credited.

\section{References}

1. Ahmad, I., Sharma, S.: Sufficiency and duality for multiobjective variational control problems with generalized $(F, \alpha, \rho, \theta)-V$-convexity. Nonlinear Anal. 72, 2564-2579 (2010) 
2. Bector, C.R., Husain, I.H.: Duality for multiobjective variational problems. J. Math. Anal. Appl. 166, 214-229 (1992)

3. Bhatia, D., Kumar, P.: Multiobjective control problem with generalized invexity. J. Math. Anal. Appl. 189, 676-692 (1995)

4. Bhatia, D., Mehra, A.: Optimality conditions and duality for multiobjective variational problems with generalized B-invexity. J. Math. Anal. Appl. 234, 341-360 (1999)

5. Ben-Israel, A., Mond, B.: What is invexity? J. Aust. Math. Soc. Ser. B 28, 1-9 (1996)

6. Caristi, G., Ferrara, M., Stefanescu, A.: Mathematical programming with $(\Phi, \rho)$-invexity. In: Konnov, I.V., Luc, D.T., Rubinov, A.M. (eds.) Generalized Convexity and Related Topics. Lecture notes in economics and mathematical systems, vol. 583, pp. 167-176. Springer, Berlin-Heidelberg-New York (2006)

7. Chen, X.H.: Duality for multiobjective variational problems with invexity. J. Math. Anal. Appl. 203, 236-253 (1996)

8. Hanson, M.A.: On sufficiency of the Kuhn-Tucker conditions. J. Math. Anal. Appl. 80, 545-550 (1981)

9. Kim, D.S., Kim, M.H.: Generalized type I invexity and duality in multiobjective variational problems. J. Math. Anal. Appl. 307, 533-554 (2005)

10. Mishra, S.K., Mukherjee, R.N.: On efficiency and duality for multiobjective variational problems. J. Math. Anal. Appl. 187, 40-54 (1994)

11. Mishra, S.K., Mukherjee, R.N.: Multiobjective control problem with $V$-invexity. J. Math. Anal. Appl. 235, 1-12 (1999)

12. Mititelu, Ş.: Efficiency conditions for multiobjective fractional problems. Appl. Sci. 10, 162-175 (2008)

13. Mititelu, Ş., Postolache, M.: Mond-Weir dualities with Lagrangians for multiobjective fractional and non-fractional variational problems. J. Adv. Math. Stud. 3, 41-58 (2010)

14. Mond, B., Chandra, S., Husain, I.: Duality for variational problems with invexity. J. Math. Anal. Appl. 134, 322-328 (1988)

15. Mond, B., Smart, I.: Duality and sufficiency in control problems with invexity. J. Math. Anal. Appl. 136, 325-333 (1988)

16. Nahak, C.: Duality for Multiobjective Control Problems With Generalized Invexity. Rendiconti Del Circolo Matematico Di Palermo Serie II, Torrto XLVII, pp. 191-206 (1998)

17. Nahak, C., Nanda, S.: Duality for multiobjective variational problems with invexity. Optimization 36, 235-248 (1996)

18. Nahak, C., Nanda, S.: On efficiency and duality for multiobjective variational control problems with $(F, \rho)$-convexity. J. Math. Anal. Appl. 209, 415-434 (1997)

19. Nahak, C., Nanda, S.: Duality for multiobjective variational problems with pseudoinvexity. Optimization 41, 361-382 (1997)

20. Preda, V.: On efficiency and duality for multiobjective programs. J. Math. Anal. Appl. 166, 365-377 (1992)

21. Reddy, L.V., Mukherjee, R.N.: Efficiency and duality of multiobjective fractional control problems under $(F, \rho)$-convexity. Indian J. Pure Appl. Math. 30, 51-69 (1999)

22. Weir, T., Mond, B.: Generalized convexity and duality in multiple objective programming. Bull. Aust. Math. Soc. 39, 287-299 (1987)

23. Xiuhong, Ch.: Duality for a class of multiobjective control problems. J. Math. Anal. Appl. 267, 377-394 (2002)

24. Zhian, L., Qingkai, Y.: Duality for a class of multiobjective control problems with generalized invexity. J. Math. Anal. Appl. 256, 446-461 (2001) 